\title{
Simulations of eccentric disks in close binary systems
}

\author{
W. Kley ${ }^{1}$, J. C. B. Papaloizou ${ }^{2}$, and G. I. Ogilvie ${ }^{2}$ \\ 1 Institut für Astronomie \& Astrophysik, Universität Tübingen, Auf der Morgenstelle 10, 72076 Tübingen, Germany \\ e-mail: wilhelm.kley@uni-tuebingen.de; kley@tat.physik.uni-tuebingen.de \\ 2 Department of Applied Mathematics and Theoretical Physics, University of Cambridge, Centre for Mathematical Sciences, \\ Wilberforce Road, Cambridge, CB3 OWA, UK
}

Received 11 April 2008 / Accepted 23 June 2008

\begin{abstract}
Context. Eccentric accretion disks in superoutbursting cataclysmic and other binary systems.

Aims. We study the development of finite eccentricity in accretion disks in close binary systems using a grid-based numerical scheme. We perform detailed parameter studies to explore the dependence on viscosity, disk aspect ratio, the inclusion of a mass-transfer stream and the role of the boundary conditions.

Methods. Using a two-dimensional grid-based scheme we study the instability of accretion disks in close binary systems that causes them to attain a quasi-steady state with finite eccentricity. Mass ratios $0.05 \leq q \leq 0.3$ appropriate to superoutbursting cataclysmic binary systems are considered.

Results. Our grid-based scheme enables us to study the development of eccentric disks for disk aspect ratio $h$ in the range $0.01-0.06$ and dimensionless kinematic viscosity $v$ in the range $3.3 \times 10^{-6}-10^{-4}$. Previous studies using particle-based methods were limited to the largest values for these parameters on account of their diffusive nature. Instability to the formation of a precessing eccentric disk that attains a quasi-steady state with mean eccentricity in the range $0.3-0.5$ occurs readily. The shortest growth times are $\sim 15$ binary orbits for the largest viscosities and the instability mechanism is for the most part consistent with the mode-coupling mechanism associated with the 3:1 resonance proposed by Lubow. However, the results are sensitive to the treatment of the inner boundary and to the incorporation of the mass-transfer stream. In the presence of a stream we found a critical viscosity below which the disk remains circular.

Conclusions. Eccentric disks readily develop in close binary systems with $0.05 \leq q \leq 0.3$. Incorporation of a mass-transfer stream tends to impart stability for small enough viscosity (or, equivalently, mass-transfer rate through the disk) and to assist in obtaining a prograde precession rate that is in agreement with observations. For the larger $q$ the location of the 3:1 resonance is pushed outwards towards the Roche lobe where higher-order mode couplings and nonlinearity occur. It is likely that three-dimensional simulations that properly resolve the disk's vertical structure are required to make significant progress in this case.
\end{abstract}

Key words. accretion, accretion disks - stars: binaries: close - novae, cataclysmic variables

\section{Introduction}

SU Ursae Majoris systems are a class of cataclysmic variable that in addition to undergoing ordinary outbursts undergo less frequent larger superoutbursts. During these, their light curves show superhumps which have a period generally slightly longer than the orbital period (for a discussion of relevant observations see for example Ak et al. 2002; Patterson et al. 2005).

A natural explanation of this phenomenon is that it is associated with a rigidly precessing eccentric accretion disk. Superoutbursts and superhumps are found to be limited to cataclysmic binaries with mass ratio $q<0.35$. Assuming that the difference between the superhump period and the orbital period represents the solid body precession of an eccentric disk, Patterson et al. (2005) discuss the establishment of the expected correlation of this period with $q$.

Studies of the light curve and effects on it associated with the mass-transfer stream impacting the disk have enabled an eccentricity of $0.38 \pm 0.1$ to be found for the disk in OY Car by Hessman et al. (1992), who also argue that the normal superhumps are produced by tidal stresses after the maximally extended disk edge approaches the companion. Similar results for IY UMa, which has a similar mass ratio, were obtained by
Rolfe et al. (2001). These authors also noted the potential importance of the impacting accretion or mass-transfer stream on the eccentric disk with its varying outer radius for understanding the late superhump light.

These observations motivate an interest in developing good numerical schemes for simulating accretion disks undergoing tidal interaction with a secondary binary component.

Although, starting with Whitehurst (1988), there have been many simulations of eccentric disks using particle-based methods (e.g. Kunze et al. 1997; Murray 1998; Smith et al. 2007, and references therein) there has been almost no grid-based work to date that is applicable to close binary systems, even though such an approach, apart from constituting a check, is promising in the context of being able to gain access to a wider region of parameter space that may be difficult to access with particle-based methods. Heemskerk (1994) performed grid-based simulations, but without including disk viscosity and was only able to obtain an eccentric disk when only the $m=3$ component of the companion's tidal potential was retained. Subsequent grid-based simulations by Stehle (1999) and Kornet \& Rozyczka (2000) also did not show clear evidence for the onset of an eccentric instability. Papaloizou (2005) solved the linearized equations for the $m=1$ mode of a free disk without a binary companion and 
considered the possible development of a parametric instability when the vertical structure was incorporated.

A study of eccentric disks also has application to other areas of astrophysics where disks and orbiting companions interact. Thus Kley \& Dirksen (2006) have studied eccentric disks in the context of the very small mass ratios appropriate to disk-planet interactions where their existence may have important consequences for theories of planet formation.

Motivated by these considerations, the aim of this paper is to make an extensive study of the dynamics of two-dimensional disks that lie in the orbital plane of a binary system, which consists of a primary star and a lower-mass secondary star in a circular orbit. The disk is centred on the primary star in a binary system with mass ratio in the range $0.05 \leq q \leq 0.3$ thought to be relevant for superoutbursting cataclysmic binaries. We perform grid-based simulations of disks that are initialised with circular motion, in order to study the onset of an eccentric instability and the properties of the resulting quasi-stationary eccentric disk. Use of a grid-based rather than a particle-based method enables consideration of disks with a wider range of viscosities and more realistic aspect ratios in the range $0.01-0.05$ that are presently difficult to access for particle-based methods such as SPH due to their inherently diffusive nature (but see Schäfer et al. 2004). We establish numerical convergence and explore the dependence of the results on the physical parameters of the disk. We also consider variations of the inner boundary conditions and the presence of a mass-transfer stream. Due to the global nature of the eccentric disk mode, results are found to be sensitive to these. When a stream is present we are able to find a transition to stability when the viscosity is reduced below a critical value. We also find that incorporation of a mass-transfer stream is important for obtaining values for the disk precession frequency that are compatible with observations.

The plan of the paper is as follows. In Sect. 2 we describe the hydrodynamic model. We then go on to describe the numerical methods we use in Sect. 2.2. Numerical simulations showing the growth of eccentricity for a typical disk model are then presented in Sect. 3. It is found that a quasi-stationary eccentric disk with mean eccentricity in the range $0.3-0.5$ is often produced. We explore the dependence of this phenomenon on disk viscosity, aspect ratio and the inner boundary condition in Sect. 4, showing that the development of an eccentric disk is favoured at high viscosity but, because it is a global phenomenon, it is sensitive to the treatment of the disk inner boundary. The quasi-stationary eccentric disk is found to precess as a rigid body. In this section we also study the dependence of the disk precession rate on the disk aspect ratio and boundary conditions showing that the precession rate is prograde for cool enough disks. We then investigate the dependence of the tendency of the disk to become eccentric on the binary mass ratio in Sect. 5 and discuss numerical resolution demonstrating the numerical convergence of our results in Sect. 6.

In Sect. 7 we explore the instability mechanism and establish the importance of the $m=3$ component of the tidal potential for causing the instability through the mode-coupling mechanism proposed by Lubow (1991a). Comparison with a linear analysis of eccentric modes is made in Sect. 8.

We go on to investigate the effect of a mass-transfer stream in Sect. 9. This is shown to make an important contribution to the disk precession rate and also to cause the disk to become stable to developing eccentricity for sufficiently small viscosity. Finally in Sect. 10 we summarize and discuss our results.

\section{Hydrodynamical model}

To model a flat two-dimensional disk we generally use a cylindrical $(r, \varphi)$ coordinate system centred on the primary star that corotates with the binary. The latter is assumed to have a circular orbit and thus to rotate uniformly. This coordinate system is convenient because the positions of the two stars remain fixed during the simulations. We have checked that the same results are obtained when simulations are run in the inertial frame. The disk material is treated as a viscous fluid with a constant kinematic shear viscosity and (except where noted below) vanishing bulk viscosity.

For a flat disk located in the orbital plane $z=0$, the velocity components are $\boldsymbol{u}=\left(u_{\mathrm{r}}, u_{\varphi}, 0\right)$. Thus $u=u_{\mathrm{r}}$ is the radial velocity and the local disk angular velocity is $\Omega=u_{\varphi} / r$ as measured in the corotating frame which rotates with the constant angular velocity, $\omega$, of the binary.

The vertically integrated continuity equation is

$\frac{\partial \Sigma}{\partial t}+\nabla(\Sigma \boldsymbol{u})=0$

The vertically integrated radial component of the equation of motion is

$$
\frac{\partial\left(\Sigma u_{\mathrm{r}}\right)}{\partial t}+\nabla\left(\Sigma u_{\mathrm{r}} \boldsymbol{u}\right)=\Sigma r(\omega+\Omega)^{2}-\frac{\partial p}{\partial r}-\Sigma \frac{\partial \Phi}{\partial r}-\Sigma b_{\mathrm{r}}+f_{\mathrm{r}}
$$

and the azimuthal component can be written in the form

$$
\frac{\partial\left[\Sigma r^{2}(\omega+\Omega)\right]}{\partial t}+\nabla\left[\Sigma r^{2}(\omega+\Omega) \boldsymbol{u}\right]=-\frac{\partial p}{\partial \varphi}-\Sigma \frac{\partial \Phi}{\partial \varphi}-r \Sigma b_{\varphi}+r f_{\varphi}
$$

Here $\Sigma$ denotes the surface density

$\Sigma=\int_{-\infty}^{\infty} \rho \mathrm{d} z$

where $\rho$ is the density, $p$ the vertically integrated (twodimensional) pressure. The gravitational potential $\Phi$ is due the primary with mass $M_{1}$ and the secondary having mass $M_{2}$ and is given by

$\Phi=-\frac{G M_{1}}{\left|\boldsymbol{r}-\boldsymbol{r}_{1}\right|}-\frac{G M_{2}}{\left|\boldsymbol{r}-\boldsymbol{r}_{2}\right|}$

where $G$ is the gravitational constant and $\boldsymbol{r}_{1}$ and $\boldsymbol{r}_{2}$ are the position vectors of $M_{1}$ and $M_{2}$, respectively. Since the mass of the disk in cataclysmic variables is only about $10^{-10} M_{\odot}$ we can safely neglect the self-gravity of the disk as well as its influence on the binary orbit. The additional terms $\boldsymbol{b}=\left(b_{\mathrm{r}}, b_{\varphi}\right)$ take into account the acceleration of the origin of the coordinate system. Rotation of the coordinate axes is taken account of through the terms involving $\omega$. The viscous force per unit area is $f=\left(f_{\mathrm{r}}, f_{\varphi}\right)$. The explicit form of the components of $\boldsymbol{f}$ is given in Kley (1999).

For simplicity we use a locally isothermal equation of state where the vertically integrated pressure $p$ is related to the surface density and sound speed through

$p=\Sigma c_{\mathrm{s}}^{2}$.

The local isothermal sound speed $c_{\mathrm{s}}$ is taken to have a fixed dependence on radius and is given by

$c_{\mathrm{s}}=\frac{H}{r} u_{\mathrm{Kep}} \equiv h u_{\mathrm{Kep}}$,

where $u_{\text {Kep }}=\sqrt{G M_{1} / r}$ denotes the Keplerian orbital velocity of the unperturbed disk that would exist in the absence of the binary companion. Equation (5) follows from vertical hydrostatic 
equilibrium. The constant ratio $h$ of the vertical height $H$ to the radial distance to the primary, $r$, is taken as a fixed input parameter. Here we adopt a standard value of

$h=H / r=0.05$.

This is somewhat large when compared to values expected for disks in cataclysmic binary systems (Smith et al. 2007), but smaller values are also considered below.

\subsection{Dimensionless units}

We adopt a system of units for which the unit of length is taken to be the orbital separation of the binary, $a_{\text {bin }}$. The unit of time, $t_{0}$, is taken to be the reciprocal of the orbital angular frequency $\omega$ of the binary. Thus

$t_{0}=\omega^{-1}=\left(\frac{a_{\text {bin }}^{3}}{G\left(M_{1}+M_{2}\right)}\right)^{1 / 2}$.

The orbital period of the binary is then given by

$P_{\text {orb }}=2 \pi t_{0}$.

The results of calculations will usually be expressed as a function of the evolution time in units of $P_{\text {orb }}$. The unit of velocity is given by $u_{0}=a_{\text {bin }} / t_{0}$. Setting the gravitational constant $G$ to be unity, Eq. (7) can be also viewed as specifying the unit of mass to be the total mass of the system $M=M_{1}+M_{2}$. The surface density can be scaled by any constant value $\Sigma_{0}$, because this drops out of the governing equations. Accordingly the surface density may be scaled to make the total disk mass be any specified value. The kinematic viscosity coefficient, $v$, is expressed in units of $v_{0}=a_{\text {bin }} u_{0}=\omega a_{\text {bin }}^{2}$.

\subsection{Numerical methods}

The governing Eqs. (1)-(3) determining the evolution of $\Sigma, u$ and $\Omega$ are solved using an Eulerian finite-difference scheme. The computational domain $\left[r_{\min }, r_{\max }\right] \times\left[\varphi_{\min }, \varphi_{\max }\right]$ is subdivided into $N_{\mathrm{r}} \times N_{\varphi}$ grid cells that are equally spaced in each coordinate direction. For typical runs we use $N_{\mathrm{r}}=200, N_{\varphi}=200$, though many have been checked at the higher resolution $N_{\mathrm{r}}=$ $300, N_{\varphi}=300$.

The numerical method utilises a staggered mesh, where scalar quantities are located at cell centres and vector quantities at cell interfaces. We adopt an operator-split scheme for which advection is based on the spatially 2nd order monotonic transport algorithm of van Leer (1977), and the viscosity can be treated either explicitly or implicitly. The basic features of the two-dimensional code RH2D that we use have been described in more detail in Kley (1989). The use of the code in cylindrical $(r, \varphi)$ coordinates has been described in Kley (1999).

Implementation in a rotating coordinate system requires special treatment of the Coriolis terms to ensure angular momentum conservation (Kley 1998). To prevent possible instabilities due to shocks, that could for example be generated by the tidal perturbation of the secondary, we use an artificial bulk viscosity as described in Kley (1999). This is in addition to the physical shear viscosity $v$. We note, that an excellent test of the correct implementation of viscosity in numerical simulations of accretion disks is the study of non-axisymmetric instabilities in the viscously spreading ring problem (Speith \& Kley 2003).

In addition, to prevent numerical instabilities due to very low densities (or very steep density gradients) near the outer boundary of the disk, where it is tidally truncated by the secondary, we introduce a "density floor", $\Sigma_{\text {floor }}$. This is applied such that whenever evolution causes the density at any location to fall below $\Sigma_{\text {floor }}$, it is reset to this value. Formally, this implementation does not conserve mass exactly, but for sufficiently low values it does not produce any influence on the dynamics. In all simulations we use a value of $\Sigma_{\text {floor }}=10^{-8}$ expressed in units such that the initial value of $\Sigma$ at $r=a_{\text {bin }}$ would be unity in the absence of tidal truncation (i.e. in units of $\Sigma_{0}$ defined in Eq. (10) below). We test the dynamical influence of the floor in Sect. 4.3 below.

Since this is an explicit code, the timestep limitation is given by the Courant condition

$\Delta t=f_{\mathrm{c}} \min _{\text {grid }} \frac{(\Delta r, r \Delta \varphi)}{|\boldsymbol{u}|+c_{\mathrm{s}}}$

where the Courant number $f_{\mathrm{c}}$ has to be smaller than one, and we typically use $f_{\mathrm{c}}=0.70$. After a few test simulations it soon became clear that, due to the large range in radius with $r_{\max } / r_{\min }$ typically larger than 10 , the required computational times become excessive because of the stringent timestep limitation arising from the inner boundary. This occurs because the Keplerian angular velocity increases rapidly as the primary star is approached according to $\Omega_{\mathrm{K}} \propto r^{-3 / 2}$. This results in approximately 25000 timesteps being required for one binary orbit with our standard resolution. As typically a few hundred orbital times need to be calculated per run, this necessitates about 10 million timesteps for each run. Exploration of a large parameter space would be prohibitive. For that purpose we implemented the FARGO algorithm (Masset 2000) into our RH2D code. In this algorithm, for each annular grid ring, the mean angular velocity $\bar{\Omega}$ is calculated and all variables are simply shifted by an integer number of grid cells, corresponding to an advection velocity as close as possible to $r \bar{\Omega}$. Advection is then only performed using the residual velocity, leading to a substantially increased timestep. This method leads, for our standard parameters using an equidistant grid, to a speed-up by a factor of about 5 .

Additional test simulations have shown that for cases that do not use the FARGO algorithm, numerical instabilities due to very small densities are likely to occur in the outer region. These could only be avoided by further reducing the timestep or adopting an implicit treatment of viscosity, both of which result in additional computational cost. A higher density floor also helps to reduce these instabilities but to avoid them entirely (and still use a large timestep) one would need unreasonably large values that possibly influence the dynamics; see the discussion on boundary conditions in Sect. 4.3 below.

Here, the use of the faster advection algorithm enhances the stability properties, allowing an explicit treatment of viscosity. A few comparison simulations that do not use FARGO, but allowed a reduced timestep, were performed to check the accuracy of our method using the NIRVANA code (Ziegler 1998). Finally, we note that the order of the applied numerical scheme matters in obtaining the eccentric state of the disk. Switching to a simple first order upwind scheme does not result in eccentric disks no matter how fine the grid resolution is chosen. For the 2nd order upwind scheme the different choices of the slope-limiter all yield very similar results.

\subsection{Setup and boundary conditions}

The coordinate system is centred on the primary star and, because it corotates with the binary system, the position of the secondary in Cartesian coordinates is fixed at $x=-1, y=$ 0 (see Fig. 1). For the radial domain we adopt $\left[r_{\min }, r_{\max }\right]$ 


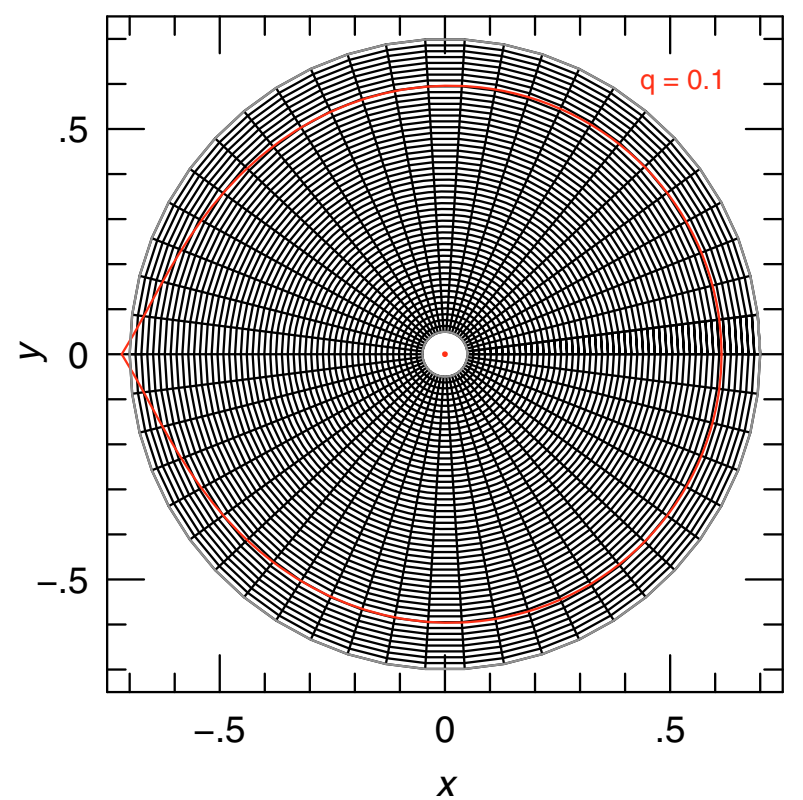

Fig. 1. The grid structure used in the computations. The radial domain is $\left[r_{\min }, r_{\max }\right]=[0.05,0.70]$ and the $\varphi$ domain is the whole annulus $[0,2 \pi]$. For our standard case this is covered with $N_{\mathrm{r}} \times N_{\varphi}=200 \times$ 200 grid cells that are equally spaced in each direction. The Roche lobe of the primary, for our standard mass ratio $q=M_{2} / M_{1}=0.1$, is defined by the additional solid (red) line. The primary and secondary star are located at $\left(x=x_{\mathrm{p}}=0, y=y_{\mathrm{p}}=0\right)$ and $\left(x=x_{\mathrm{s}}=-1, y=y_{\mathrm{s}}=0\right)$ respectively. Note that in this figure, for illustrative purposes, we display a low-resolution $50 \times 50$ grid.

where $r_{\text {min }}=0.05$ and $r_{\max }=0.70$ for all models in units where the binary separation is unity. The azimuthal domain is taken to be $[0,2 \pi]$.

For the initial surface density profile, for $r<0.4$ in dimensionless units, we adopt a power law of the form

$\Sigma(r, \varphi)=\Sigma_{0} r^{-1 / 2}$.

For $r>0.4, \Sigma$ is reduced smoothly towards a minimum value of $10^{-8} \Sigma_{0}$. The initial velocity is taken to be pure Keplerian rotation around the primary star as would be measured in an inertial frame $\left(u_{\mathrm{r}}=0, r \Omega=\left(G M_{1} / r\right)^{1 / 2}-r \omega\right)$. No correction is made for pressure effects or the non-axisymmetric binary potential. The presence of the secondary star is allowed to cause the surface density distribution to evolve away from the initial profile on a dynamical timescale. This normally causes a rapid adjustment of the outer profile through tidal truncation of the disk. The fixed temperature stratification is always given by $T(r) \propto r^{-1}$ which follows from the assumed constant aspect ratio $h=H / r=$ const.

In the azimuthal $(\varphi)$ direction, periodic boundary conditions for all variables are imposed. At the outer radial boundary of the grid which extends somewhat beyond the Roche lobe of the primary (see Fig. 1) we use standard outflow boundary conditions. This imposes zero gradient conditions on all variables apart from the radial velocity, i.e. the values in the ambient ghost cells have the same values as the last active grid point. The radial velocity at the ambient ghost cell is set equal to the value at $r=r_{\max }$ if the flow direction is outward (zero gradient condition) and zero otherwise. This outflow condition allows material to leave the system but inflow is not permitted.

As we found that the form of the boundary condition applied at the inner boundary may play an important role in determining
Table 1. Parameters of the standard model. Values are quoted in dimensionless units.

\begin{tabular}{ll}
\hline \hline Mass ratio $\left(q=M_{1} / M_{2}\right)$ & 0.1 \\
Disk thickness $(h=H / r)$ & 0.05 \\
Viscosity $(v)$ & $10^{-5}$ \\
Radial range $\left(\left[r_{\min }, r_{\max }\right]\right)$ & {$[0.05,0.70]$} \\
Grid $\left(N_{\mathrm{r}} \times N_{\varphi}\right)$ & $200 \times 200$ \\
Inner boundary & reflecting \\
\hline
\end{tabular}

the characteristics of the flow, we implemented four different boundary conditions at $r_{\min }$ :

- Rigid reflecting: here the inner boundary acts as a solid wall where the radial velocity is set to zero, while for the other variables mirror symmetry conditions are applied to determine values in ghost cells.

- Viscous outflow: here an axisymmetric form for the radial velocity at the inner boundary is specified. This is directed inward with a magnitude given by

$u\left(r_{\min }\right)=-\frac{3}{2} \frac{v}{r_{\min }}$.

This boundary condition imposes a steady-state accretion disk flow.

- Damping condition: all variables within a certain region near the inner boundary are relaxed on the orbital timescale at $r_{\min }$ towards their initial values. This approach reduces wave reflection from the inner boundary and is described in de Val-Borro et al. (2006). In addition $u_{\mathrm{r}}\left(r_{\mathrm{min}}\right)$ is fixed at zero for all time.

- Free outflow: this boundary condition corresponds to the outflow condition applied at $r_{\max }$. The radial flow velocity at $r_{\min }$ can only be directed inwards and no inflow from the inner regions into the computational domain is allowed.

We found from our numerical simulations that the form of the inner boundary condition can make quite a difference in the results concerning the eccentricity and precession rate of the disk.

\section{Standard model}

To study the effect of varying the physical and numerical parameters defining the simulations, we first consider in detail a standard model for use as a reference for the extensive parameter studies we have performed. The parameters defining this model are summarized in Table 1.

We now describe the characteristic behaviour of the standard model. Due to the strong gravitational perturbation induced by the secondary, the initial axisymmetry of the disk is rapidly destroyed and a strong two-armed spiral density response is formed. This is shown in Fig. 2 where the surface density is displayed after the system has evolved for one orbital period of the binary. The two-armed spiral which is strongest in the outer disk region but extends all the way through the disk down to the primary star is clearly visible. The surface density shows some evidence for a non-axisymmetric pattern near the inner boundary of the disk, confined to the innermost two radial gridcells. Such a feature (here a $m=6$ mode) is generated typically in gridbased simulations with reflecting inner boundary conditions, and is not a consequence of using the FARGO-algorithm. It can be reduced or minimised by using special, non-reflecting or damping, boundary conditions. After the onset of the eccentric instability this feature dissapears in the simulation. 


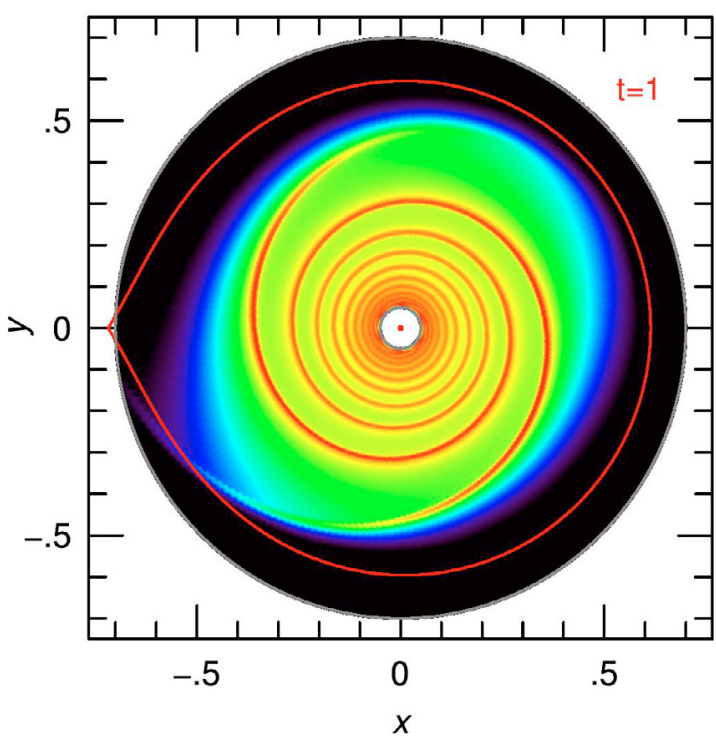

Fig. 2. Greyscale plot of the surface density of the disk for the standard model after 1 binary orbit. The solid (red) curve indicates the Roche lobe of the primary star (central red dot).

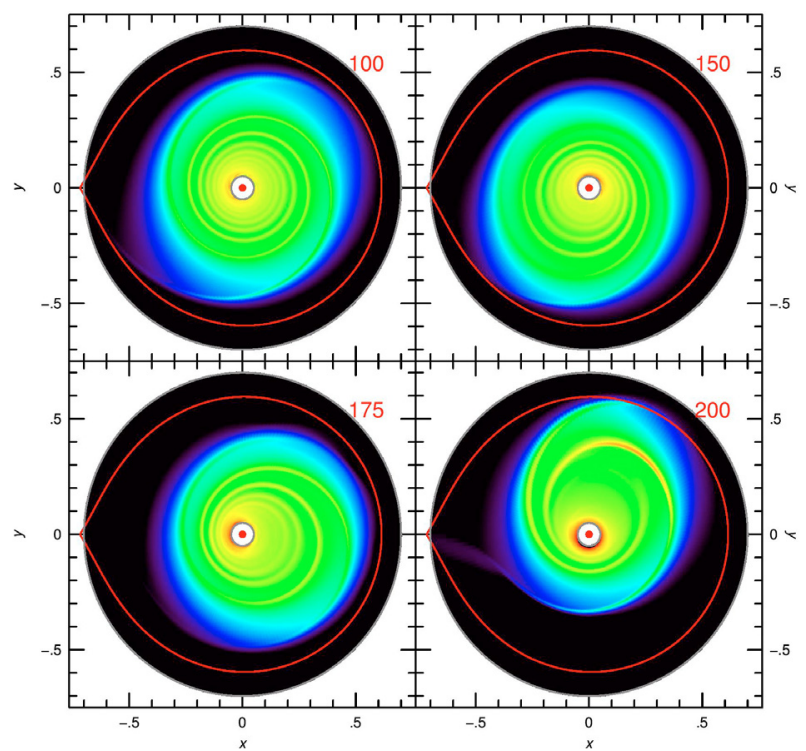

Fig. 3. Greyscale plot of the surface density of the disk for the standard model at 100,150,175 and 200 orbital periods. The solid (red) curve indicates the Roche lobe of the primary star (central red dot).

In the subsequent evolution the disk slowly becomes eccentric. This is indicated in Fig. 3 where we display the twodimensional surface density structure at four different times. The distortion of the disk is evident at later times. While in the first snapshot taken after 100 orbits there is only a slight indication of disk eccentricity, the subsequent panels demonstrate a growing eccentric disk mode and one can infer the presence of an apsidal line joining the locations of minimum and maximum displacements from the primary. Additionally there is indication for precession of this apsidal line.

The tidal torques induced by the secondary also lead to a truncation of the disk and a rearrangement of the (mean) radial surface density profile. This is demonstrated in Fig. 4 where the azimuthally averaged density is shown at 5 different times. The solid black curve indicates the initial (axisymmetric) profile, $\Sigma=r^{-1 / 2}$, with a taper applied for $r>0.4$. Clearly, the

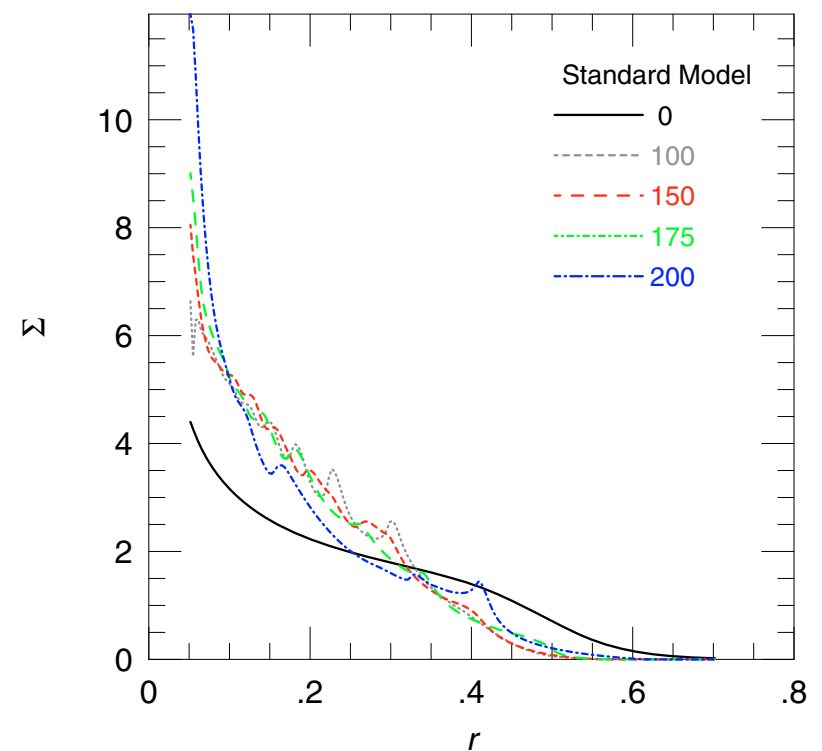

Fig. 4. Radial distribution of the azimuthally averaged surface density $\Sigma(r)$ at different times. The solid black curve indicates the tapered initial profile.
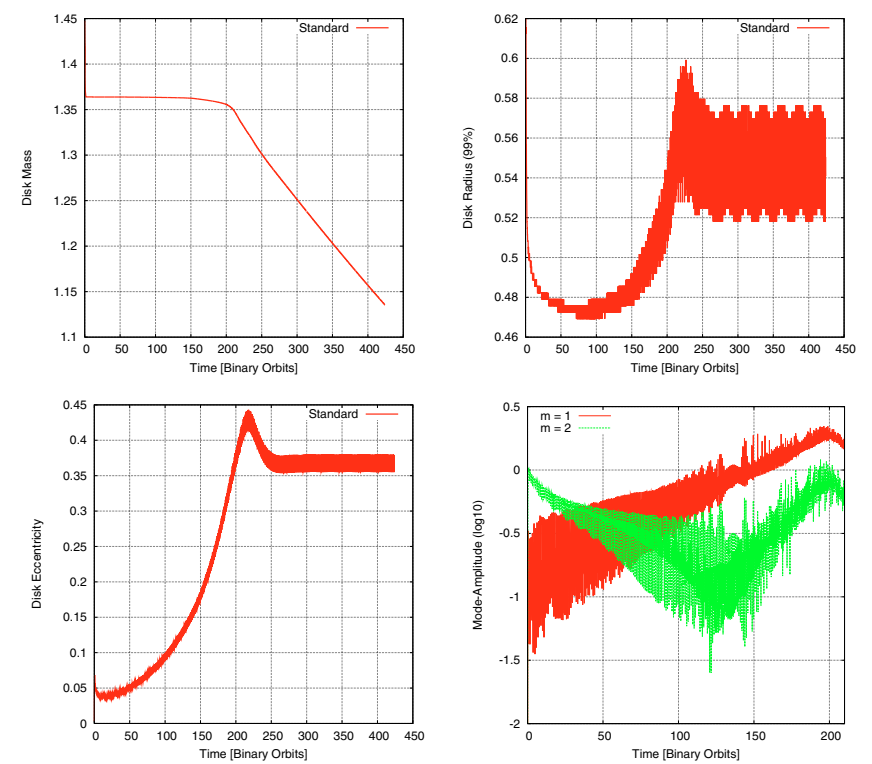

Fig. 5. Evolution of various global disk properties of the standard model as functions of time. The total mass in the computational domain, or equivalently the disk mass, is shown in the upper left panel. The disk radius, defined as the radius containing $99 \%$ of the mass, is shown in the upper right panel. The disk eccentricity is shown in the lower left panel and the $m=1$ and $m=2$ Fourier amplitudes are shown in the lower right panel.

disk becomes truncated at a radius around $r \approx 0.5$ where the surface density starts to drop much more steeply. After about 250 orbits the average surface density distribution attains a new quasi-stationary profile (see below).

In Fig. 5 we display the time-evolution of several global disk quantities (in dimensionless units). The total mass in the computational domain is seen to drop off rapidly within the first 2 orbits due to the initial mass loss through the outer boundary induced by the tidal perturbation of the secondary (see also Fig. 2). After this initial burst of mass loss, the disk is tidally truncated, such that as seen in Fig. 5, the disk radius, defined to be the radius 
containing $99 \%$ of the mass, is reduced from 0.61 to 0.48 . This is then smaller than the Roche lobe. The mass subsequently remains constant until $t \approx 200$. The disk radius reaches a minimum at around $t \approx 80$, and increases slowly thereafter. This increase of the radius of the disk is coupled with an increase in the mean eccentricity $e_{\mathrm{d}}$ of the disk which is displayed in the bottom left panel of Fig. 5. The quantity $e_{\mathrm{d}}$ is determined by first calculating the eccentricity for each individual gridcell assuming that it is in an unperturbed orbit around the central star, with the position and velocity given by the actual grid values averaged to the cell centres. To obtain the global value $e_{\mathrm{d}}$ we then perform a massweighted average over the individual cell values. The longitude of the disk's periastron $\varpi_{\mathrm{d}}$ is calculated in the same way. The explicit formulae are given for example in Murray \& Dermott (1999).

As seen in Fig. $5, e_{\mathrm{d}}$ begins to exponentiate after $t \approx 20$ with an $e$-folding time of about 80 orbits. After about 250 orbits the eccentricity has settled to a final value of $e_{\mathrm{d}}=0.37$ with an average outer disk radius $r_{\mathrm{d}} \approx 0.55$. The increase in $e_{\mathrm{d}}$ is also associated with a further decrease in the disk mass. Beyond $t \approx 250$ mass spills over the Roche lobe and is lost from the system. This mass loss does not affect the dynamics of the system (i.e. it does not change the equilibrium values of $e_{\mathrm{d}}$ and $r_{\mathrm{d}}$ ) because the absolute value of the mass density scales out of the evolution equations. This feature has been confirmed numerically in additional test calculations where the total mass in the system is kept constant by rescaling the density of all grid cells at each timestep in order to account for the lost mass. In the bottom right panel of Fig. 5 the time-evolution of the $m=1$ and $m=2$ surface density Fourier amplitudes calculated at a single disk radius $(r=0.37)$ are displayed. The $m=1$ mode associated with the eccentricity increases exponentially from the beginning of the run up to $t \approx 200$ after which induced additional mass loss leads to a reduction in the amplitude. To show the evolution more clearly we have plotted here only the first 200 orbits. The only other Fourier amplitude that plays a significant role is that associated with $m=2$, which at early times (when the spiral arm feature dominates the disk) is much larger than the $m=1$ amplitude, but it becomes weaker at later times. In the final stages it increases again, saturating eventually at a value still smaller than the $m=1$ amplitude.

As mentioned above, Fig. 3 indicates global precession of the eccentric disk. This is quantified in Fig. 6 where the solid (red) curve corresponds to the time-evolution of the longitude of pericentre (periastron) of the disk, $\varpi_{\mathrm{d}}$, calculated in the same manner as $e_{\mathrm{d}}$. Obviously the disk precesses as a whole at a constant rate, a feature that is supported by the additional light (green) curve which corresponds to the phase of the $m=1$ Fourier component at one given radius $r=0.37$. Both angles precess at the same rate, confirming our interpretation. The periodic disturbances in $\varpi_{\mathrm{d}}$ coincide with the times when the disk's apocentre is closest to the secondary star. In this case the outer parts of the eccentric disk are strongly disturbed which renders the calculation of $\varpi_{\mathrm{d}}$ more inaccurate. From the plot, which shows the phases in the inertial frame, we conclude that the disk experiences a slow retrograde precession with a period $P_{\mathrm{d}} \approx 16.4$ orbits.

To study the internal structure of the disk we show in Fig. 7 the disk's radial distribution of eccentricity and the longitude of pericentre at different times (indicated by the legend) which lie within the time interval plotted in Fig. 6. The distribution of eccentricity within the disk does not vary much with the binary orbital phase and drops smoothly towards zero in the centre of the disk, due to the rigid boundary at the inner radius. In the

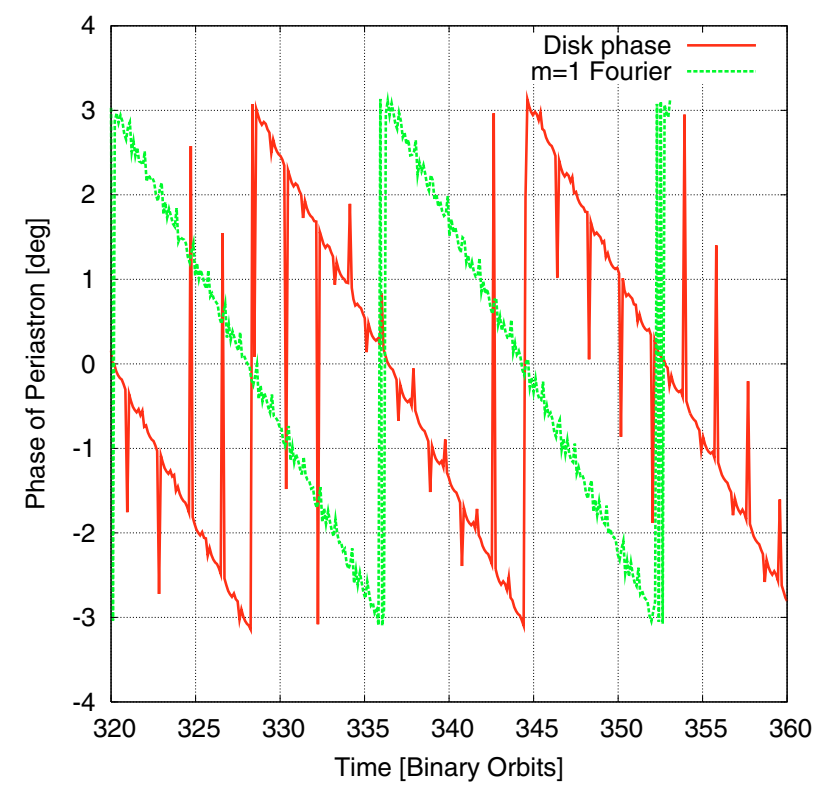

Fig. 6. Time-evolution of the longitude of pericentre (periastron) of the disk. The solid darker (red) curve is the mass-weighted mean pericentre over the whole disk, calculated as described in the text. The lighter dashed (green) curve corresponds to a phase of the $m=1$ Fourier mode at the single radius $r=0.37$. Note that the phases are calculated in the inertial frame.
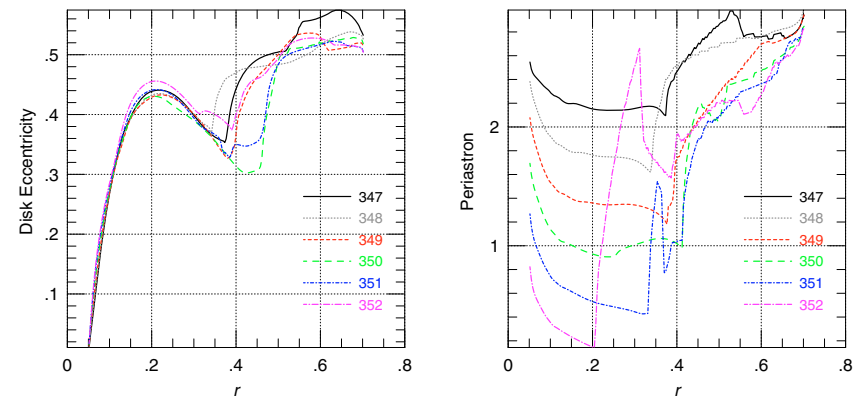

Fig. 7. Radial variation of the eccentricity and longitude of pericentre of the disk at various times (quoted in binary orbits).

main part of the disk the eccentricity lies around $e=0.4$ which is consistent with two-dimensional surface plots of the density (see the snapshot at $t=200$ in Fig. 3). The radial variation of the pericentre indicates a small twist within the innermost parts of the disk, which is larger when the disk's apocentre is closest to the secondary star (at times 351 and 352) when it can reach about 45 degrees. In addition, the fact that the curves at different times appear vertically shifted indicates again the precession of the disk as a single entity.

\section{Dependence on viscosity, temperature and the inner boundary condition}

\subsection{Viscosity}

The first parameter we investigate is the kinematic viscosity $v$. To study dependence of the results on the magnitude of the kinematic viscosity we started from our standard model $\left(v=10^{-5}\right)$ and varied only the value of $v$ keeping all other parameters unchanged.

The variation of the mean disk eccentricity with time is displayed for different kinematic viscosities in Fig. 8, where the 


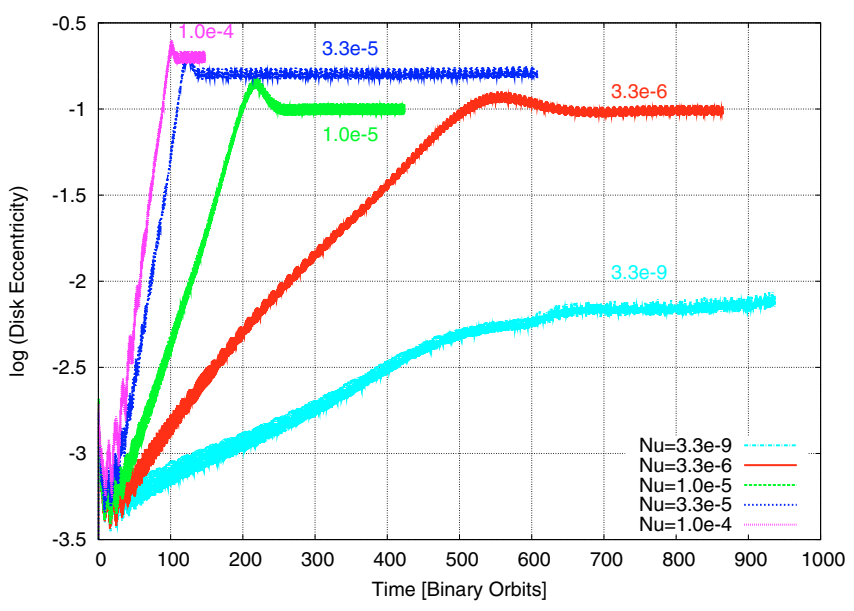

Fig. 8. Time-evolution of the mean eccentricity of the disk (natural logarithm) for different kinematic viscosities (in dimensionless units).
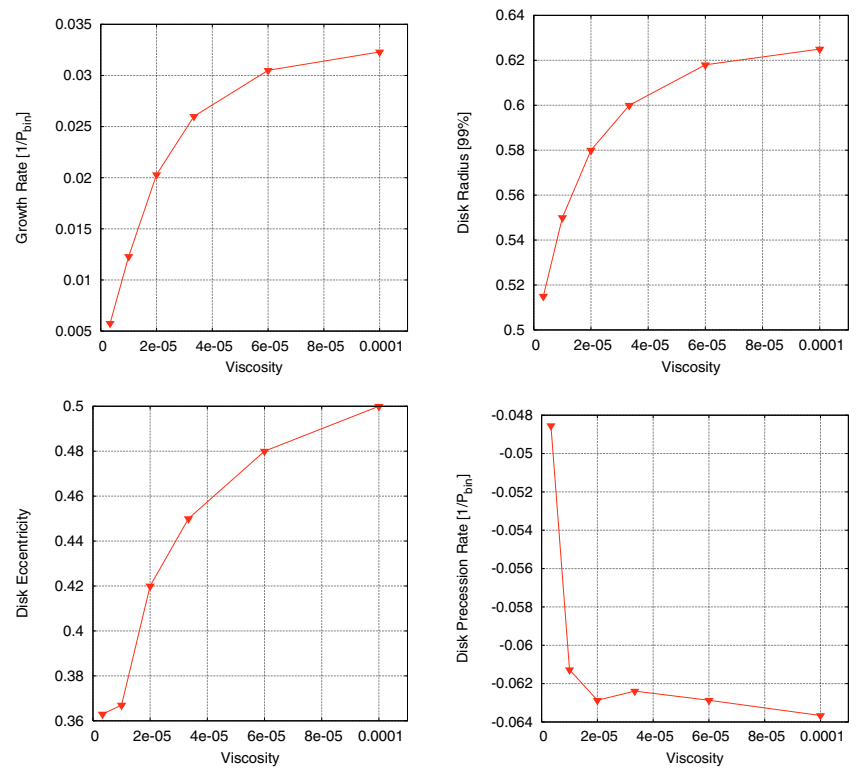

Fig. 9. Dependence of various global disk parameters on the magnitude of the viscosity. upper left panel: growth rate of the eccentricity of the disk; upper right panel: the radius, $r_{\mathrm{d}}$, within which $99 \%$ of the mass of the disk is contained; lower left panel: the mass-weighted mean eccentricity of the disk $e_{\mathrm{d}}$; lower right panel: precession rate of the disk $\dot{\varpi}_{\mathrm{d}}$, negative values indicating retrograde precession. The values for $r_{\mathrm{d}}, e_{\mathrm{d}}$ and $\dot{\varpi}_{\mathrm{d}}$ are determined at a time at which the system reaches its final quasi-steady state.

middle (green) curve refers to the standard model which (for reference) has been displayed already in Fig. 5 at early times. The growth rate of the eccentricity depends strongly on the magnitude of the viscosity: increasing the viscosity shortens the growth time substantially, while for smaller viscosities it takes much longer for the disk to become eccentric. For the lowest-viscosity model $\left(v=3 \times 10^{-9}\right)$, which corresponds essentially to an inviscid calculation, the disk does not attain significant eccentricity in a run time of 1000 orbits. However, there is an indication that there may be a very long-term eccentricity growth produced by numerical effects. From the upper left panel of Fig. 9 a simple extrapolation indicates a limiting value of $v$ for which the disk becomes eccentric of $v \sim 2 \times 10^{-6}$. Already the growth time for $v=3.3 \times 10^{-6}$ is $\sim 162$ orbits. In general the models settle to a quasi-stationary state with a constant eccentricity, which

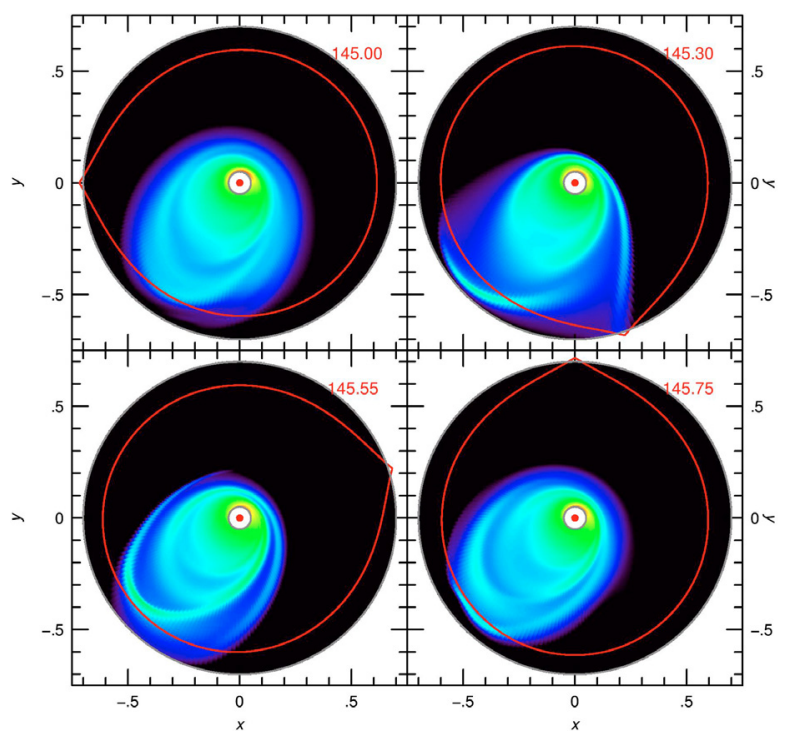

Fig. 10. Four snapshots of the disk surface density contours during one orbital period as viewed in the non-rotating frame. Time increases from top to bottom and left to right The solid (red) curve indicates the Roche lobe of the primary star. The viscosity is $10^{-4}$, the mass ratio is 0.1 and $H / r=0.05$. As the companion has a close approach to the disk outer edge a tidal tail is pulled out (top right). This subsequently rejoins the disk (bottom left) which remains relatively unperturbed until the next close approach.

decreases as the viscosity decreases. However, quasi-steady states with mean eccentricities below $e=0.36$ do not seem to be reached for this model by decreasing the viscosity alone. But then a longer time is required for this value to be attained.

Clearly the initial growth of $e_{\mathrm{d}}$ in these models is exponential and growth rates, $\sigma_{\mathrm{d}}$, defined through

$e_{\mathrm{d}}(t) \propto \exp \left(\sigma_{\mathrm{d}} t\right)$

can be determined by fitting simulation data to the above relation. The results are plotted in the upper left panel of Fig. 9 where the variation of $\sigma_{\mathrm{d}}$ with viscosity is plotted. In the remaining 3 panels we plot the equilibrium values of the radius $r_{\mathrm{d}}$, the eccentricity $e_{\mathrm{d}}$ and the precession rate $\dot{\varpi}_{\mathrm{d}}$ of the disk. Each of the quantities $\left(\sigma_{\mathrm{d}}, r_{\mathrm{d}}, e_{\mathrm{d}}\right)$ shows a strikingly similar dependence on $v$. This behaviour can be understood in terms of the diffusive influence of viscosity. For larger viscosity the disk material will tend to spread outwards at a faster rate with more material being pushed to the outer regions of the disk where it feels a stronger (eccentricity enhancing) tidal perturbation due to the secondary. Hence the increase of the growth rate with viscosity. For the very same reason, the equilibrium value of the radius $r_{\mathrm{d}}$ as well as the final eccentricity will increase with $v$. The fact that all these quantities respond similarly to variations of $v$ indicates that they respond in a similar manner to the outward expansion of the disk. On the contrary, the disk precession rate $\dot{\varpi}_{\mathrm{d}}$ depends only weakly on the viscosity (for $v \gtrsim 5 \times 10^{-6}$ ). From the lower two panels $\left(e_{\mathrm{d}}, \dot{\varpi}_{\mathrm{d}}\right)$ there is an indication that for $v \leq 10^{-5}$ the disk shrinks rapidly causing corresponding changes to the precession rate while the eccentricity levels off. However, we did not consider simulations with $v<3.3 \times 10^{-6}$ as it is likely that at this stage they become resolution-limited such that the numerical viscosity cannot be neglected.

Finally we illustrate the behaviour of the eccentric disk once it has attained its final quasi-steady state for the case with $v=10^{-4}$ in Fig. 10. Cases with other values of $v$ show similar 

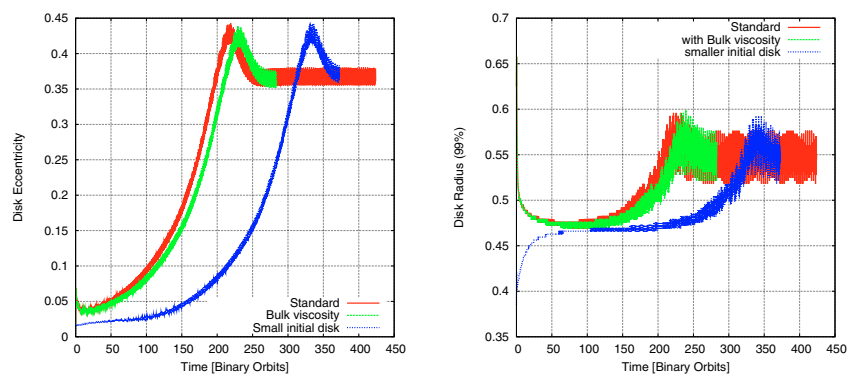

Fig. 11. Mean disk eccentricity $e_{\mathrm{d}}$ and disk radius $r_{\mathrm{d}}$ as functions of time for the standard model (red curve), the same case but with an added explicit bulk viscosity $v_{\text {bulk }}=2 v$ (green curve) and a case with standard parameters, but for which the initial disk radius is taken to be $r_{\mathrm{d}}(0)=0.3$ (blue curve).

behaviour. In Fig. 10 we show the surface density contours during one orbital period as viewed in the non-rotating frame. In this frame the secondary (moving counter clock wise) has a close approach to the slowly precessing disk's apocentre approximately once every orbit. As the companion approaches the disk's outer edge a tidal tail is pulled out. This subsequently rejoins the disk which remains relatively unperturbed until the next close approach. As even at apocentre, the disk matter rotates faster than the binary, so the tidal tail leads the secondary and is clearly responsible for angular momentum transfer to the orbit. This must also be associated with enhanced tidal dissipation. As the disk appears relatively unperturbed away from these closest approaches, most of the tidal angular momentum transfer may occur as a result of these close approaches.

\subsubsection{Effect of bulk viscosity}

The imposed kinematic viscosity, $v$, is intended to represent the effects of some form of disk turbulence, probably arising from the magnetorotational instability (Balbus \& Hawley 1998). It is possible that an effective bulk viscosity may also result which could also act as a stabilizing mechanism. In addition, in typical SPH simulations on this problem an explicit bulk viscosity is included; see for example the recent study by Smith et al. (2007). In order to investigate its effects, we performed runs for which an explicit bulk viscosity $\zeta=\Sigma v_{\text {bulk }}$ was added. In this study we used a constant $v_{\text {bulk }}$ and present in Fig. 11 results for the case when the bulk viscosity coefficient is twice as large as the shear viscosity coefficient: $v_{\text {bulk }}=2 v$. The influence of the added bulk viscosity on the simulations is very small: the only effect is a slightly increased damping, as indicated for example by the longer growth time. Otherwise an added bulk viscosity has no dynamical influence. This indicates that the direct effect of viscosity on the eccentric mode is unimportant and implies that mechanisms such as viscous overstability cannot be responsible for the eccentric instability seen here. We also plot the evolution for a case where the initial disk radius $r_{\mathrm{d}}(0)=0.3$, rather than the usual $r_{\mathrm{d}}(0)=0.5$, in Fig. 11. As expected the initial growth is slowed down due to the small outer disk radius which remains around $r_{\mathrm{d}}=0.47$ until $t=150$. Only when the eccentric instability, which appears to grow (though more slowly) even for this small disk, has developed sufficiently does the radius of the disk increase again. (It should be noted that, owing to the way in which it is defined, an increase of $r_{\mathrm{d}}$ may be associated purely with a growth of the eccentricity of the disk rather than an increase of the semimajor axis at which the disk is tidally truncated.) At later times the behaviour is in fact identical to the

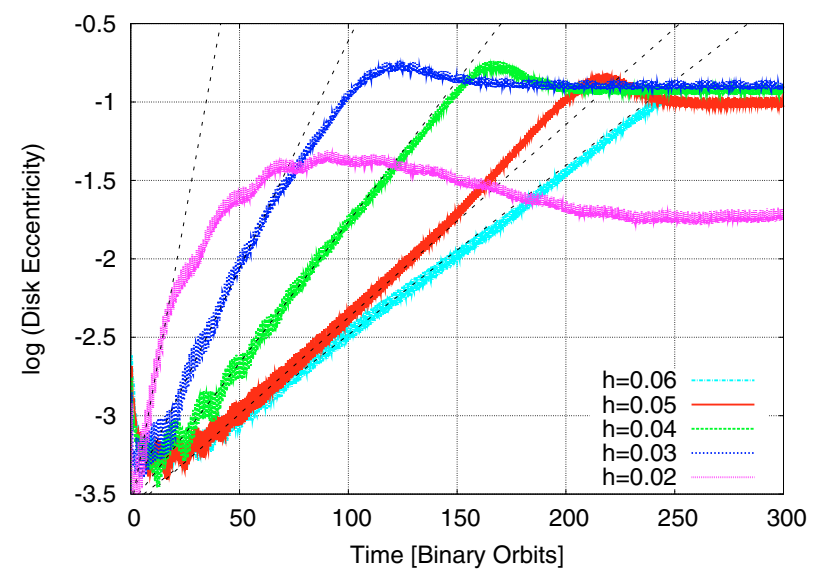

Fig. 12. Time-evolution of the mean eccentricity of the disk for different disk aspect ratios $h \equiv H / r$.
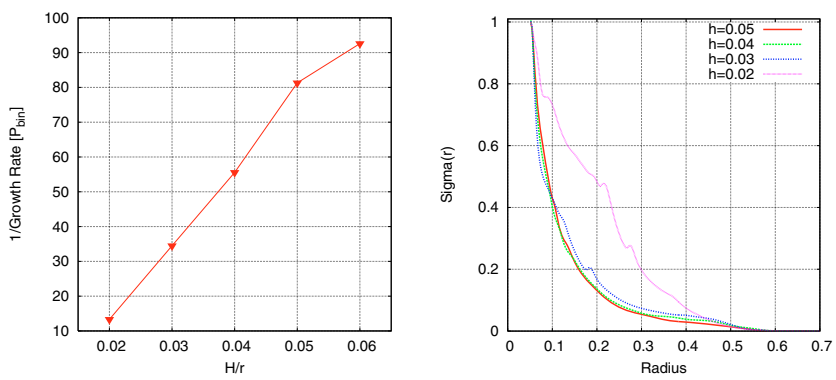

Fig. 13. Left: dependence of the growth time $\left(\sigma_{\mathrm{d}}^{-1}\right)$ of disk eccentricity on $h=H / r$. Quoted is the time (in orbital periods) to increase the eccentricity by a factor of $e$; Right: the equilibrium surface density profile of the disk normalized to the central value for different $h$.

standard model. Hence, different initial conditions lead to the same outcome, as one might expect for viscous flows.

\subsection{Temperature}

In this section we study the effect of variations of the disk temperature or, equivalently, the relative scaleheight $h=H / r$ of the disk. Starting again from the standard model we vary $h$ from 0.01 to 0.06 . The variation of $e_{\mathrm{d}}(t)$ is displayed in Fig. 12, where the thick (coloured) lines refer to different values of $h$, and the dashed lines are eyeball fits made during the initial growth period. The growth timescale depends on the disk temperature and it is found to be shorter for cooler disks. The final disk eccentricities do not depend on the value of $h$ as long as $h \geq 0.03$. For the smallest value displayed here, $h=0.02$, the standard grid resolution $(200 \times 200)$ is not sufficient. In additional simulations we found that runs (for $h=0.02$ ) with higher resolution yield larger values of $e_{\mathrm{d}}$ more consistent with the other runs. The final radius of the disk, $r_{\mathrm{d}}$, also does not depend on the disk aspect ratio for $h>0.02$. All runs yield an outer disk radius $r_{\mathrm{d}} \approx 0.55$, with a slight tendency for larger disks to be associated with larger $h$.

The inferred growth times as a function of $h=H / r$ are plotted in the left panel of Fig. 13. The growth is fastest for cooler disks and the growth time $1 / \sigma_{\mathrm{d}}$ increases linearly with increasing $h$. For the thinnest disks we study with $h=0.02$, the $e$-folding time is around 10 orbits (for the standard viscosity). In the right panel of Fig. 13 the equilibrium surface densities are displayed for various $h$. Obviously the value of $h$ does not play a role in determining the equilibrium structure. At higher 


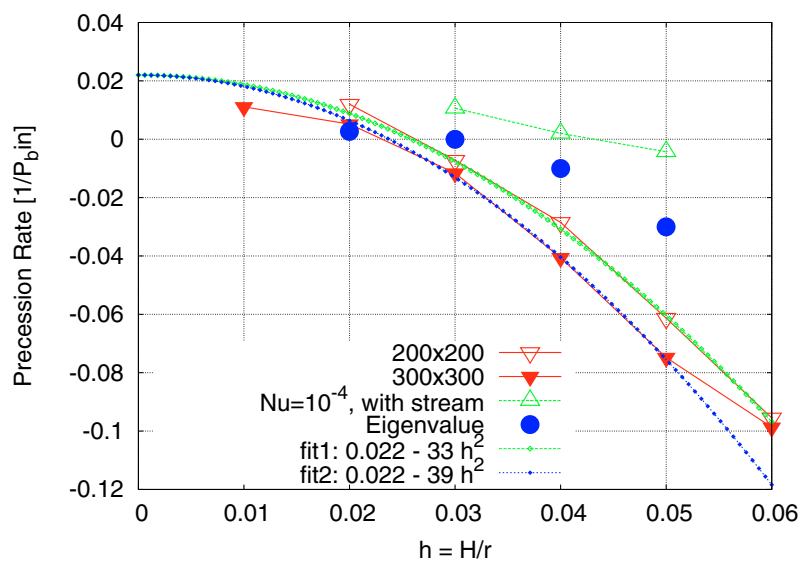

Fig. 14. Dependence of the equilibrium disk precession rate $\dot{\varpi}_{\mathrm{d}}$ on disk temperature. The two separate lines with the triangles refer to two different grid resolutions, the upper to the standard case and the lower to $300 \times 300$. The additional curves with the small open and solid diamonds are approximate parabolic fits to the data points. Values obtained by solving the linear eigenvalue problem using the azimuthally averaged surface density profiles are also indicated by the filled circles. The open triangles are obtained from models where the disk is impacted by a mass-transfer stream (see Sect. 9 below).

resolution, the surface density profile of the cool $h=0.02$ disk is in better agreement with the others as well.

We plot the equilibrium precession rate of the disk as a function of $h$ in Fig. 14. The inverse of this quantity gives the period of one complete turn of the disk (in the inertial frame). Values obtained by solving the linear free $m=1$ mode eigenvalue problem using the mean surface density distributions shown in Fig. 13 and assuming a free outer boundary are also plotted (for a discussion of this approach see Papaloizou 2005; or Goodchild \& Ogilvie 2006). These are in good agreement with the results derived from the nonlinear simulations. For very cool disks, $h \lesssim 0.025$, the precession rate is positive while for hotter disks the disk precesses in a retrograde sense. The precession period at the transition is infinite, corresponding to the disk being stationary in the inertial frame. In general the precession rate is determined by a combination of different effects. There is a contribution, $\dot{\varpi}_{\text {dyn }}$, due to the axisymmetric component of the binary potential, and there is also a pressure contribution, $\dot{\varpi}_{\text {press. }}$ The first part $\dot{\varpi}_{\text {dyn }}$ is positive, giving a prograde contribution, while the latter part $\dot{\varpi}_{\text {press }}$ is negative, tending to produce retrograde precession. Our results confirm the existence of these contributions and indicate a parabolic dependence of $\dot{\varpi}_{\mathrm{d}}$ on $h$ for locally isothermal disks. The influence of the viscosity on the precession rate is negligible as we have indicated above.

A change of the direction of precession sometimes occurs during the earlier growth phases of the simulations and is illustrated in Fig. 15 where we plot the time-evolution of the apsidal line of the disk for $h=0.03$. Initially, during the period of eccentricity growth, the disk precesses in a prograde sense and at time $t \approx 130$, as it settles into a quasi-stationary state, it turns around and undergoes retrograde precession. Thus this value of $h$ lies close to the borderline separating these two possibilities for the final state.

We also remark that the precession rate is sensitive to the mass distribution in the outer regions of the disk and it can be changed from retrograde to prograde by supplying matter from the secondary through the $L_{1}$ point (see Fig. 14 and Sect. 9 below).

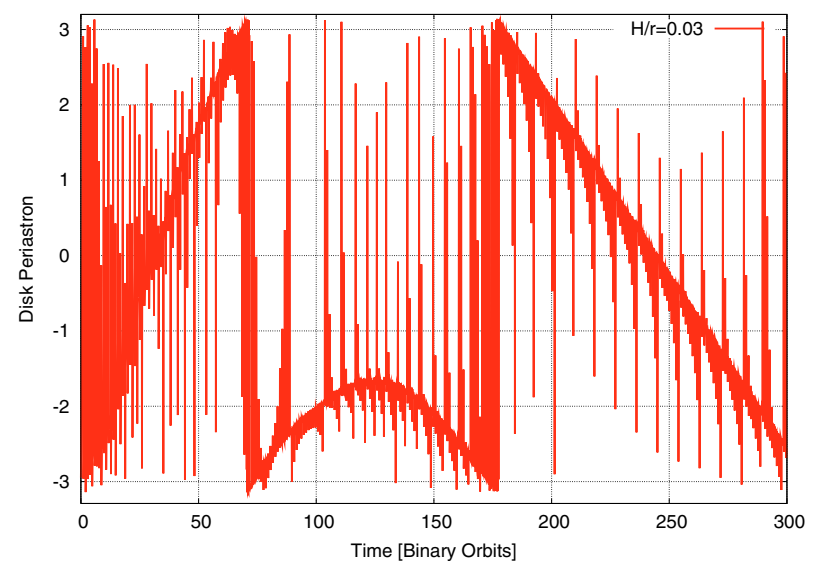

Fig. 15. Time-evolution of the pericentre (in the inertial frame) of the disk, $\varpi_{\mathrm{d}}(t)$, for a model with an intermediate $H / r=0.03$.

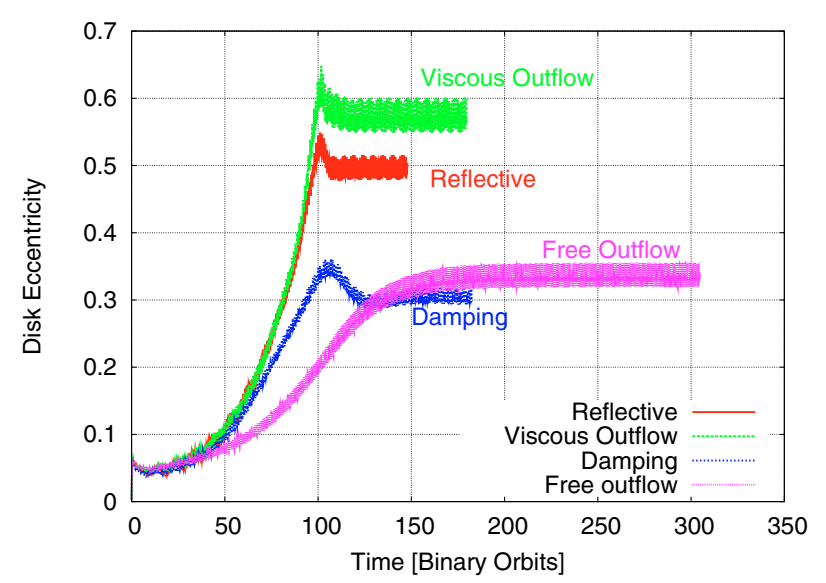

Fig. 16. Time-evolution of the mean eccentricity of the disk for different inner boundary conditions for a kinematic viscosity of $v=10^{-4}$ (ten times the standard value).

\subsection{Effect of the inner boundary condition}

We here discuss the effects of the different kinds of boundary conditions that we have implemented as described in Sect. 2.3, and discuss briefly the influence of a higher density floor. The evolution of the mean eccentricity of the disk for the different inner boundary conditions for a kinematic viscosity of $v=10^{-4}$ is shown in Fig. 16.

The results shown in Fig. 16 indicate that the adoption of an inner boundary condition with damping properties (such as the damping boundary conditions and the outflow condition) lead to configurations that differ from the standard case. Both the growth rate and the mean equilibrium eccentricity of the disk are strongly affected. Increased damping yields smaller growth rates and smaller final $e_{\mathrm{d}}$. The outer disk radius shows similar tendency but it varies within a much smaller range from $r_{\mathrm{d}} \approx 0.60$ for the damping boundary condition to $r_{\mathrm{d}} \approx 0.64$ for the viscous outflow condition (see Table 2). The viscous outflow condition and the standard reflecting condition behave very similarly because both have strong reflective properties. The most pronounced influence of the boundary is on the precession rate of the disk, as is indicated in Table 2. For the free outflow condition we find a very slow positive, i.e. prograde, precession of the disk. However, as is indicated in Fig. 17, a very pronounced eccentric inner hole forms in the centre of the disk which does not happen in cases where the inner boundary is forced to be axisymmetric. When an open boundary condition is used, 
Table 2. Results obtained using the different inner boundary conditions and density floor.

\begin{tabular}{llll}
\hline \hline Boundary condition & $e_{\mathrm{d}}$ & $r_{\mathrm{d}}$ & $\dot{\varpi}_{\mathrm{d}}\left[1 / P_{\text {orb }}\right]$ \\
\hline Reflecting & 0.50 & 0.640 & -0.064 \\
Viscous outflow & 0.56 & 0.651 & -0.060 \\
Damping & 0.31 & 0.598 & -0.046 \\
Free Outflow & 0.33 & 0.597 & +0.0037 \\
Reflecting, high floor & 0.52 & 0.620 & -0.022 \\
\hline
\end{tabular}

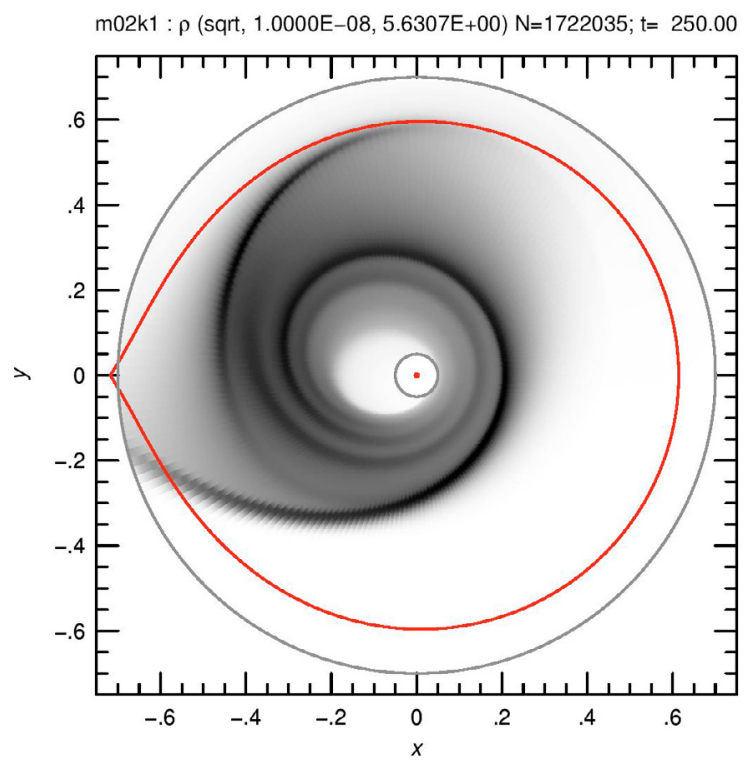

Fig. 17. Two-dimensional surface density structure for the model with the open outflow inner boundary condition after 250 orbital periods.

material on an eccentric orbit can leave the system freely and is not "reflected back" into the computational domain. A sideeffect of the use of the open boundary condition (and also the damping condition) is a reduction in the overall eccentricity of the disk. Clearly, the treatment of the inner boundary condition is an important issue that needs to be considered in more detail. In the case of a central compact stellar object, such as a white dwarf or neutron star, which has a very small pressure scaleheight in its upper layers, it might behave approximately as a rigid wall with typical reflecting wall conditions for the normal velocity and a no-slip boundary condition for the tangential velocity. This is the situation of the boundary layer problem in the theory of accretion disks which has an associated radial lengthscale that is too small to make inclusion tractable in a simulation of the type considered here. However, it appears that the overall disk properties may be significantly affected by the behaviour of this small innermost region of the disk. The final line in Table 2 refers to a model with a reflecting inner boundary and a high density floor of $10^{-3}$ (instead of $10^{-8}$ ). While the final values of the disk's eccentricity and radius are not changed much, there is a significant difference in the precession rate. The higher density in the outer parts of the primary's Roche lobe slows down the precession by a factor of about 2.8 . Hence, to accurately determine the important rate of disk precession a small floor value is clearly required.

\section{Mass ratio}

We now investigate the influence of the mass ratio $q=M_{2} / M_{1}$ on the disk dynamics. To speed up the simulations all of the runs

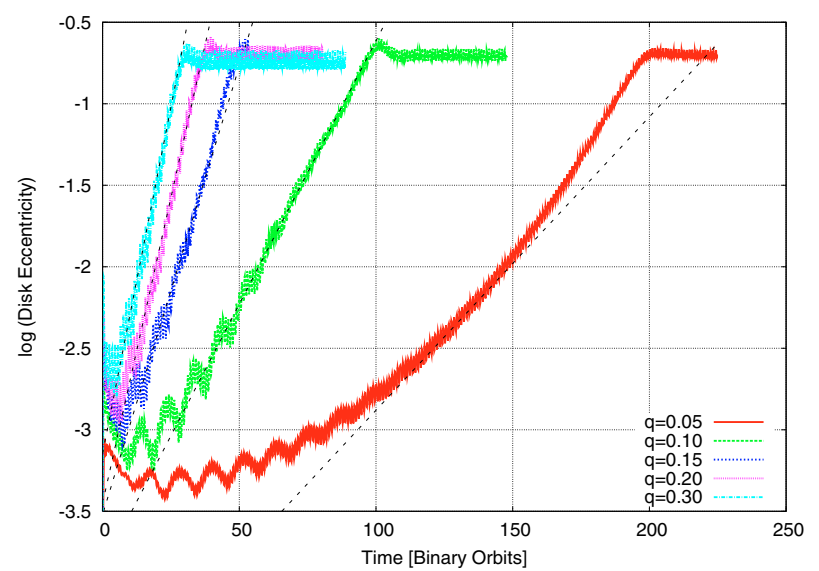

Fig. 18. Time-evolution of the mean eccentricity of the disk (logarithmic) for different mass ratios $q=M_{2} / M_{1}$ of the binary for a kinematic viscosity of $v=10^{-4}$ (ten times the standard value). The thinner black dashed lines indicate approximate linear fits.
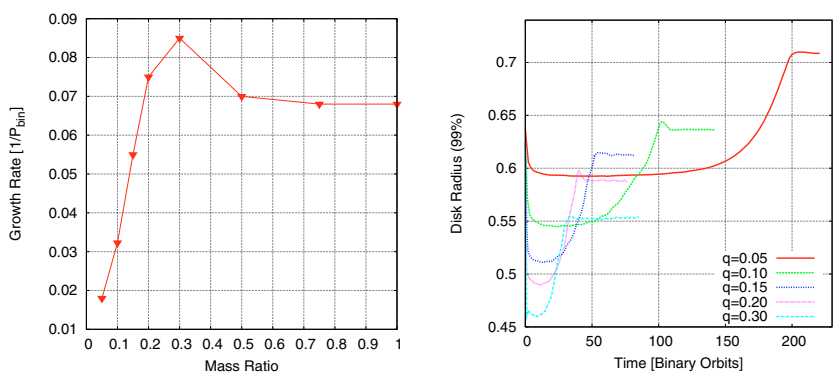

Fig. 19. Left: dependence of the growth rate $\left(\sigma_{\mathrm{d}}\right)$ of the disk eccentricity on the mass ratio q. Right: the disk radius $r_{\mathrm{d}}$ as a function of time for different $q$. The curves are smoothed.

in this section have been performed with $v=10^{-4}$ which is ten times larger than adopted for the standard simulation (see Table 1).

The eccentricity as a function of time is shown in Fig. 18 for different $q$ with over-plotted straight-line fits indicating exponential growth. For larger mass ratios $q$ the growth is strongly enhanced, but all models settle to approximately the same final eccentricity $e_{\mathrm{d}}$. For the smallest mass ratio $(q=0.05)$ there is no direct indication of an exponential growth phase and we have drawn an approximate straight-line fit applicable to the middle of the growth phase. The extracted growth rates are plotted as a function of mass ratio in the left panel of Fig. 19. For small $q$ the growth rate increases linearly with $q$ but it slows down at the largest value $q=0.3$ plotted. We have performed explorative runs for larger $q$ and find eccentricity growth all the way to $q=1$ with fastest grow occuring at $q \approx 0.3$ (see Fig. 19). Below it is argued that this behaviour may be related to higher order non-linear mode coupling which becomes more important for larger $q$, but in this work we do not elaborate on this issue any further.

The variation of the disk radius with time (right panel of Fig. 19) shows a sharp drop in $r_{\mathrm{d}}$ just after the start of the simulations due to the tidal effect of the secondary which is stronger for larger $q$. After attaining a minimum value, as low as 0.46 for the largest $q$, the disk expands again on the eccentricity growth timescale. The quasi-stationary disk radius is smaller for larger $q$ due to the enhanced tidal effects. Note that for the $q=0.05$ case we have used a larger outer boundary radius $r_{\max }=0.77$. 

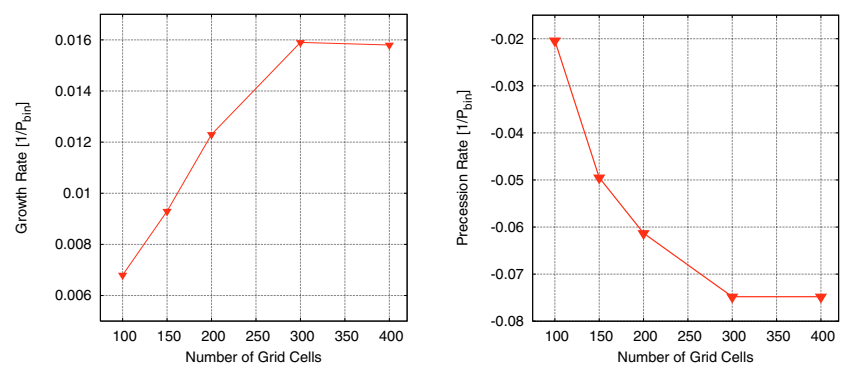

Fig. 20. Left: dependence of the growth rate $\left(\sigma_{\mathrm{d}}\right)$ of the disk eccentricity on the numerical resolution. The two-dimensional grid always has an equal number of grid points in each direction. Thus in the standard case 200 corresponds to $200 \times 200$. Right: the precession rate of the disk as a function of resolution.

\section{Numerical resolution}

An important issue in (multi-dimensional) simulations is the question of numerical resolution. To study its effect we have varied the grid resolution in our standard model from $100 \times 100$ up to $400 \times 400$. The grids we use are always uniform. The models are typically started at $t=0$ to study the whole growth phase of the evolution. In some cases higher-resolution simulations have been started using results obtained from coarser grids to provide initial data with the object of investigating the quasi-stationary state. Our main results are contained in Fig. 20 where the dependence of the growth and precession rates of the disk are indicated for different grid resolutions. The physical parameters used are those of the standard model. At low resolution, the growth rate increases approximately as a linear function of the number of grid cells. Beyond $300 \times 300$ it levels off which indicates that we have reached numerical convergence. This finding is corroborated by the fact that the precession rate apparently levels off at the same resolution (right panel). The converged growth rate $\sigma_{\mathrm{d}} \approx 0.016$ differs from the value for our standard resolution $\left(\sigma_{\mathrm{d}} \approx 0.012\right)$ by approximately $30 \%$; similarly the value of $\dot{\varpi}_{\mathrm{d}}$ differs by about $25 \%$. While the absolute values for the growth and precession rate change, the qualitative behaviour of the disk, in particular the prograde slow precession in this case, does not change.

\section{Instability mechanism}

\subsection{Mode coupling}

In this section we investigate the mechanism of excitation of the disk eccentricity. A mechanism responsible for this that involves the coupling of velocity (or surface density) perturbations with different azimuthal mode numbers through the companion's tidal potential has been described by Lubow (1991a). This was later confirmed by Lubow (1991b) by carrying out and analysing appropriate SPH simulations.

Although incorporating the slow precession of the disk as viewed in the inertial frame in the discussion below would introduce minor adjustments, for simplicity we neglect it. Then when viewed from this frame, the eccentricity of the disk may be considered to be associated with a stationary surface density perturbation with azimuthal mode number $m=1$. A disk in which the gas orbits in circles about the primary is subject to a tidal potential with components which vary as trigonometric functions of $m(\varphi-\omega t), m=0,1,2,3, \ldots$ These produce corresponding responses in the state variables that scale with the mass ratio, $q$.
When the disk becomes eccentric through the excitation of an $m=1$ mode that is approximately stationary in the inertial frame, nonlinear coupling between the response to the tidal potential and this mode produces an effective forcing which will be a sum of components that are trigonometric functions of $(m \pm 1) \varphi-m \omega t, m=0,1,2,3 \ldots$ These will have amplitudes that scale as $e q, e$ being the characteristic disk eccentricity. More generally a coupling of order $j$ produces effective forcing $(m \pm j) \varphi-m \omega t, m=0,1,2,3 \ldots$ with pattern speed $m \omega /(m \pm j)$ and amplitude scaling as $e^{j}$.

\subsection{Energy and angular momentum exchange with the companion}

Each of these effective forcings produces a surface density response that results in energy and angular momentum exchange with the companion. This exchange may in some cases produce a feedback causing the eccentricity to grow. This works because a companion in fixed circular orbit must undergo angular momentum changes, $\Delta J$, and energy changes, $\Delta E$, such that $\Delta J=\Delta E / \omega$. However, the angular momentum and energy changes associated with a disturbance with pattern speed $m \omega /(m \pm j)$ are such that $\Delta J=\Delta E(m \pm j) /(m \omega)$. Thus for surface density disturbances with the - alternative, that rotate faster than the companion and are thus expected to transfer orbital angular momentum to it, too little is transferred per unit of energy transferred. The difference has to come from the disk orbits which accordingly become eccentric. Energy and angular momentum transfer to the companion is expected to be especially effective if there is an associated Lindblad resonance in the disk (Goldreich \& Tremaine 1980).

Similarly disturbances with the + alternative, which rotate more slowly than the companion and are expected to gain angular momentum, may cause eccentricity growth. However, such disturbances are less closely matched to the rotation of the disk and in particular have no Lindblad resonances within it (Goldreich \& Tremaine 1979) and are thus not expected to be significant.

Accordingly, disturbances rotating with pattern speeds $m \omega /(m-j)$, with $m$ and $j \neq m$ being integers, may be associated with eccentricity growth if there is an associated significant energy and angular momentum transfer. The presence of a Lindblad resonance is expected to enhance this. Although in principle any value of $j$ might contribute to eccentricity growth, linear instability could result only when $j=1$ (Lubow 1991a) and the forcing as a result of coupling is linear in $e$. In that case the only value of $m$ leading to a disturbance with a Lindblad resonance within the disk is $m=3$. From the above discussion this disturbance has azimuthal mode number $m-j=k=2$ and pattern speed $3 \omega / 2$. The associated Lindblad resonance corresponds to the 3:1 resonance at which the disk orbital period is one third of that of the binary. When this is present the mode coupling may lead to a linear instability to eccentricity growth (Goodchild \& Ogilvie 2006).

According to the above view of the mode coupling leading to eccentricity growth, the surface density perturbation corresponding to the disk eccentricity should grow together with a surface density perturbation with $k=2$ and angular frequency $l \omega$, with $l=3$. This disturbance is derived from a coupling between the disk eccentricity and the $m=3$ component of the tidal potential, which accordingly plays an important part in the process. 


\subsection{Mode amplitudes}

Following Lubow (1991b), in order to demonstrate the operation of this mechanism and the significance of the $m=3$ component of the tidal potential, we define the mode amplitude associated with the pair $(k, l)$ as

$S_{k l}=\sqrt{\left(a_{k l}^{2}+b_{k l}^{2}+c_{k l}^{2}+d_{k l}^{2}\right)}$.

In this expression

$$
\begin{aligned}
& a_{k l}=\int_{t}^{t+2 \pi} \int_{A} \Sigma\left(r, \varphi, t^{\prime}\right) \cos (k \varphi) \cos \left(l \omega t^{\prime}\right) \mathrm{d} A \mathrm{~d} t^{\prime} \\
& b_{k l}=\int_{t}^{t+2 \pi} \int_{A} \Sigma\left(r, \varphi, t^{\prime}\right) \sin (k \varphi) \sin \left(l \omega t^{\prime}\right) \mathrm{d} A \mathrm{~d} t^{\prime} \\
& c_{k l}=\int_{t}^{t+2 \pi} \int_{A} \Sigma\left(r, \varphi, t^{\prime}\right) \cos (k \varphi) \sin \left(l \omega t^{\prime}\right) \mathrm{d} A \mathrm{~d} t^{\prime} \\
& d_{k l}=\int_{t}^{t+2 \pi} \int_{A} \Sigma\left(r, \varphi, t^{\prime}\right) \sin (k \varphi) \cos \left(l \omega t^{\prime}\right) \mathrm{d} A \mathrm{~d} t^{\prime},
\end{aligned}
$$

where the integration is over the surface area of the disk, $A$. Note that $S_{k l}$ also involves integration over the domain $(t, t+2 \pi)$ and is thus a function of the local time $t$.

In order to test the predictions of the above mode-coupling analysis, we evaluate the time-evolution of the mode amplitudes for the $(k, l)$ pairs $(1,0),(1,3),(2,3),(3,3),(2,2),(3,2)$ for runs with $q=0.1$ and $q=0.2$. We describe below results obtained with the kinematic viscosity $v=10^{-4}$. However, similar results are obtained for $v=10^{-5}$. In order to establish the importance of the component of the tidal potential with $m=3$, we consider cases for which the full tidal potential is used, only the $m=3$ component of the tidal potential is used and finally for which the full tidal potential with the $m=3$ component removed is used. By studying the time-evolution of the mode amplitudes in these cases, the importance of the $m=3$ component of the tidal potential could be confirmed. Note that because the defined mode amplitudes involve integrals over the disk radius, the significance of their absolute values is unclear because of cancellation effects. However, they may still be used to relate to the growth rates of linear modes.

The time-evolution of the mode amplitudes $S_{k, l}$ for $q=0.1$ is plotted in Fig. 21. The upper panel gives results for the case when only the component of the tidal potential with $m=3$ is retained. The lower panel gives results corresponding to the case when the full tidal potential is used. In both cases the mode with $k=2$ and $l=3$ grows at the same rate as the mode with $k=1$ and $l=0$, the latter mode being responsible for the disk eccentricity. Although it is difficult to demonstrate the causal nature of this connection, this behaviour would certainly be expected from the discussion of mode coupling given above. We also find that the final state and magnitude of the eccentricity of the disk is similar in all cases for which the disk is unstable to becoming eccentric. However, the growth rate is measured to be smaller when the full potential is used. Figure 22 gives similar plots to those shown in in Fig. 21 but for $q=0.2$. Note that, as expected from the mode-coupling theory, there is a very close correspondence between the mode with $k=2$ and $l=3$ and the mode with $k=1$ and $l=0$ when only the $m=3$ component of the tidal potential is used for this mass ratio.
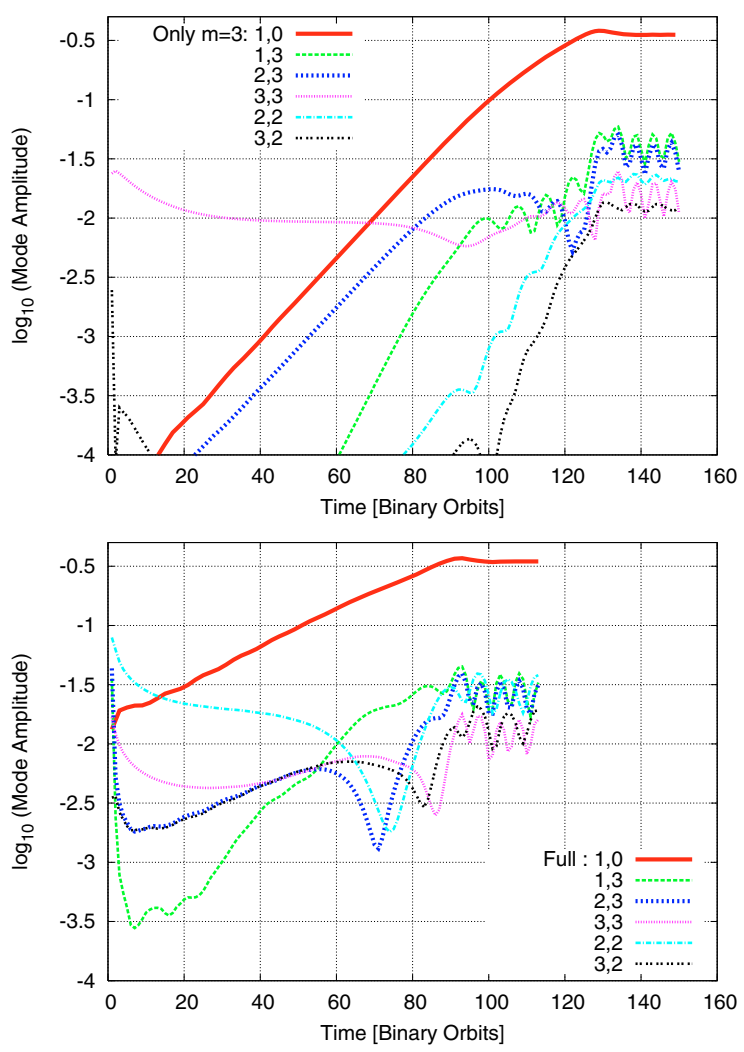

Fig. 21. Time-evolution of the mode amplitudes $S_{k, l}$ (see Eq. (13)) for $q=0.1$ with a kinematic viscosity $v=10^{-4}$ plotted on a logarithmic scale. The upper panel gives results for the case when only the component of the tidal potential with $m=3$ is retained. The lower panel gives results corresponding to the case when the full tidal potential is used. In both cases the mode with $k=2$ and $l=3$ grows at the same rate as the mode with $k=1$ and $l=0$, the latter mode being responsible for the disk eccentricity. The growth is slower when the full potential is used.

\subsection{Effectiveness of the component of the tidal potential with $m=3$}

In order to determine whether the $m=3$ component of the tidal potential is essential for producing eccentricity growth, we performed simulations using the full tidal potential with the $m=3$ component removed. The time-evolution of the mode amplitudes $S_{k, l}$ is shown in Fig. 23 for $q=0.1$ and $q=0.2$. When $q=0.1$ little growth of the disk eccentricity is seen confirming the importance of the $m=3$ component. However, when $q=0.2$ some growth is seen but at a significantly slower rate than when the full potential is used.

Although the results discussed above indicate the importance of the $m=3$ component of the tidal potential, they also indicate that it is not always needed. From Fig. 23 it is apparent that although eccentricity growth is very small when $q=0.1$, it is still present but reduced when $q=0.2$. The time required to attain saturation is increased from about 30 to 150 orbits in that case.

As in the other cases the mode with $k=2$ and $l=3$ grows at the same rate as the mode with $k=1$ and $l=0$ indicating that this is still responsible for causing eccentricity growth. Also a response with $k=3$ and $l=3$ is still present even though it cannot be driven by the $m=3$ component of the potential. The most natural explanation is that this response is produced by a forcing originating from a coupling between the response to the dominant $m=2$ tide and the $m=1$ tide. As before that can then couple to the $S_{1,0}$ mode producing eccentricity growth. Thus in 

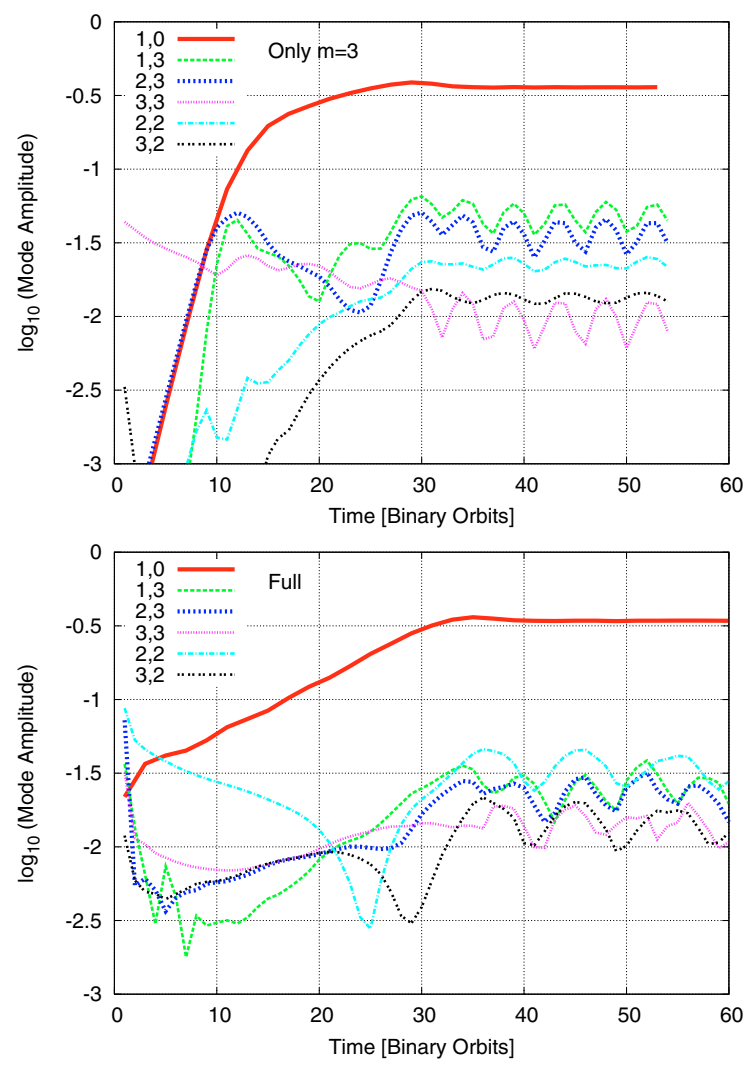

Fig. 22. As in Fig. 21 but for $q=0.2$. Note that there is a very close correspondence between $S_{2,3}$ and $S_{1,0}$ when only the $m=3$ component of the tidal potential is used.

this case one has replaced the $(3,3),(1,0)$ pairwise coupling by a $(2,2),(1,1),(1,0)$ triple coupling. In so doing the strength is reduced by a factor $q$ making the process less significant in the smaller mass ratio case as is seen in the simulations. This process thus contributes more effectively for larger $q$. On the other hand the 3:1 resonance which enables effective angular momentum transport between disk and companion is pushed towards the Roche lobe requiring further disk expansion and resulting in the process becoming increasingly nonlinear.

\section{Comparison with linear theory}

In an attempt to understand the linear phase of the simulations, during which the eccentricity is small and grows exponentially in time, we analysed the full set of linear equations governing small perturbations, with azimuthal mode number $m=1$, of a steady, axisymmetric disk. (This is in addition to the approach mentioned in Sect. 4.2, in which approximations are made that are appropriate for slowly evolving global eccentric modes.) For the purposes of comparison with the standard model in which a reflecting inner boundary condition is used, the basic state of the linear calculation is a non-accreting solution with zero radial velocity.

This approach yields appropriate solutions corresponding to slowly precessing eccentric modes of the disk, of which the "lowest-order" mode with the simplest radial structure might be expected to be the most relevant. The precession rate can be positive or negative depending on the mass ratio of the binary, the value of $H / r$, the surface density profile and the boundary conditions. While it is possible, using this approach, to account for a number of aspects of the simulation results, the sensitivity of
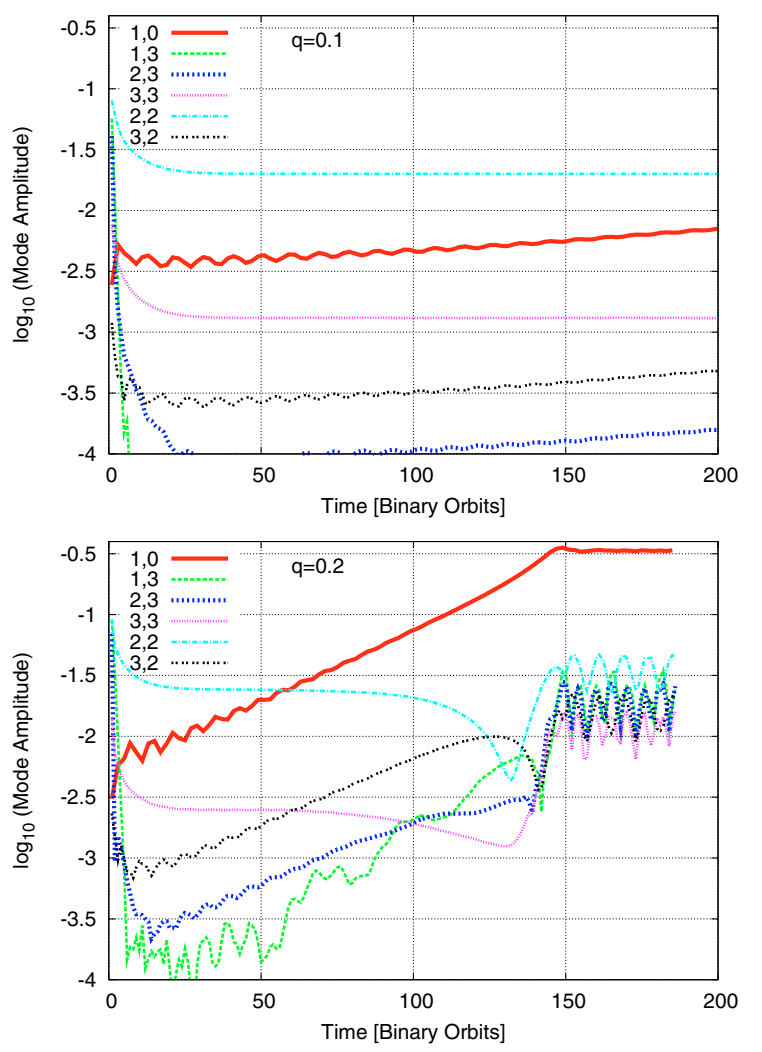

Fig. 23. Time-evolution of the mode amplitudes $S_{k, l}$, plotted on a logarithmic scale, for $q=0.1$ (upper panel) and $q=0.2$ (lower panel). For these cases, the tidal potential with the $m=3$ component removed is used and the kinematic viscosity $v=10^{-4}$. When $q=0.1$ little growth of the disk eccentricity or $S_{1,0}$ is seen. When $q=0.2$ some growth occurs but at a significantly slower rate than when the full potential is used (see Fig. 22).

the precession rate to the exact surface density profile and the boundary conditions is a matter of concern.

Such modes also grow or decay slowly as a result of viscosity. It is known that shear viscosity can render modes unstable through the mechanism of viscous overstability (Kato 1978), in which some of the energy being drawn by viscous stresses from the differential rotation of the disk is diverted into growing oscillations. Viscous overstability has been identified as an important issue in the theory of eccentric disks (Ogilvie 2001). A local analysis in the case of an isothermal two-dimensional disk with constant kinematic shear viscosity and no bulk viscosity indicates overstability for radial wavelengths $\gtrsim 9 H$ and a maximum local growth rate of approximately $0.034 \mathrm{v} / \mathrm{H}^{2}$ for a radial wavelength of approximately $13 H$ (Latter \& Ogilvie 2006). This behaviour is confirmed in our global linear calculations. For sufficiently thin disks, growing modes are obtained. However, for the standard model with $H / r=0.05$, the preferred local wavelength of the viscous overstability is as long as $0.66 r$ and in fact we do not find overstable global modes. In addition, we confirm by this method that the inclusion of a bulk viscosity with $v_{\text {bulk }}=2 \times 10^{-5}$ makes a negative contribution to the growth rate that is quite negligible in comparison with those found in the simulations. Therefore viscous overstability is not relevant to the standard model but might be an important issue when thinner disks are considered.

It is possible within this linear approach to model the nonlinear mode-coupling process by adding terms that correspond to a localised growth of the eccentric perturbations in the vicinity of 
the 3:1 resonance. Rather than localising the growth in the form of a delta function as done by Goodchild \& Ogilvie (2006), this effect can be represented using a Gaussian or Lorentzian function of radius centred on the resonance and with a width appropriate to a Lindblad resonance broadened by pressure. Including these terms allows us to obtain growing eccentric modes with growth rates comparable to those obtained in the simulations. However, it is again found that a detailed comparison is difficult because of the sensitivity of the results to the exact surface density profile and the treatment of the resonance.

According to the angular momentum equation in the case of a uniform shear viscosity, the surface density in the nonaccreting basic state should deviate from the initial $r^{-1 / 2}$ profile only as a result of the tidal torque applied to the disc. Papaloizou \& Pringle (1977) provide a method to calculate the tidal torque per unit radius, which gives a result proportional to the viscosity of the disk. When this is balanced with the viscous torque it predicts a surface density profile that is independent of the viscosity and depends only on the mass ratio $q$. Unfortunately this approach does not give accurate agreement with the surface density profile shown in Fig. 4, indicating that the angular momentum balance is nonlocal and involves propagating density waves not considered by Papaloizou \& Pringle (1977). The standard model disk is therefore smaller than predicted by Papaloizou \& Pringle (1977). We note that a two-dimensional isothermal disk may allow radial wave propagation to a greater extent than more realistic models. It may also be relevant that SPH simulations are less likely to allow these waves to propagate and may therefore produce larger disks that are more susceptible to eccentric instability. Clearly the tidal truncation of disks needs to be better understood in order to make accurate predictions of the growth and precession rates of eccentric modes.

\section{Effect of a mass-transfer stream}

To make better contact with possibly more realistic cases with mass overflow from the secondary we have performed some studies where we modify the outer boundary condition to allow for mass inflow through the $L_{1}$ point. The model parameters are dealt with in the same way as before. We adopt the mass ratio $q=0.1$ which allows a scaling to be made such that binary parameters match very closely those of OY Car which has a primary of mass $M_{1}=0.685 M_{\odot}$ and $P_{\text {orb }}=0.063 \mathrm{~d}$. The physical separation of the two stars is thus $a_{\text {bin }}=0.627 R_{\odot}$. Mass is injected into the system at a constant rate. Note that in the case of locally isothermal disk models with constant kinematic viscosity as adopted here, the independence of the dynamics of the flow, and in particular the final values of eccentricity and disk radius in a quasi-steady state, to the surface density scale means that the results are independent of the magnitude of the inflow rate! This may thus be scaled to a value appropriate for cataclysmic variables, typically $\dot{M}=10^{-9} M_{\odot} /$ yr. However, in a more realistic disk model that includes suitable radiation and energy transport mechanisms and allows for the possibility of time-dependent outbursts, this would not be the case.

In the simulations presented here the Roche lobe of the primary is as empty as possible initially, i.e. the density is constant and equals the floor value.

The first set of simulations were performed with the standard viscosity $v=10^{-5}$ in dimensionless units which would scale to a value of $2.2 \times 10^{13} \mathrm{~cm}^{2} / \mathrm{s}$ for the OY Car system. The inner boundary condition is open to allow for accretion of mass onto the primary star and enable a steady state to be reached. We have performed simulations with both the "viscous outflow" and the
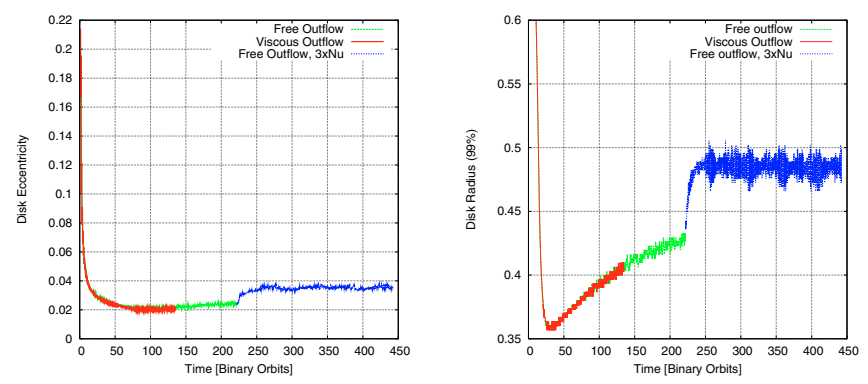

Fig. 24. Mean disk eccentricity $e_{\mathrm{d}}$ and disk radius $r_{\mathrm{d}}$ as functions of time for models that include mass inflow through the $L_{1}$ point and different inner boundary conditions and viscosities. For the first two plots (green and red curves) the standard viscosity $10^{-5}$ has been used, while the third plot (blue curve) is for a model that has been continued from the first at time $t \approx 230$ but with $v=3 \times 10^{-5}$.

"free outflow" conditions as described in Sect. 2.3 above. From Fig. 24 it is clear that the standard viscosity value used does not result in an eccentric disk. Even an increase of the viscosity by a factor of 3 does not produce an eccentric disk in the "free outflow" case as the final (blue) curve in Fig. 24 indicates. In this latter case the disk's radius is increased to $r_{\mathrm{d}} \approx 0.48$ due to the spreading action of viscosity but the disk nevertheless does not switch to an eccentric mode. The apparently large initial disk radii in these models are explained by the fact that the disk is initially empty and the $99 \%$ radius is contributed to by the material that is infalling from the $L_{1}$ point.

We then ran models with a larger viscosity $v=10^{-4}$ using the "viscous outflow" condition at the inner boundary. The models were started from both an empty Roche lobe and a standard initial disk as in the previous simulations. In these cases the disk attained an eccentric mode. In both cases an identical final state is reached, however much more quickly for the model with an initial disk. The evolution of the total mass in the disk (within the primary's Roche lobe) is displayed in the top left panel of Fig. 25. The two cases show a complementary behaviour: in the case with an initial disk the total mass drops towards the final equilibrium (at around $\log m_{\text {disk }}=-4$ ) while for the empty case it rises initially, overshoots the equilibrium point and settles to the equilibrium value roughly 100 orbits later. Additional disk properties such as the disk radius, eccentricity and precession rate are all independent of the initial condition as indicated by the other panels in Fig. 25.

\subsection{Disk dynamics}

The dynamical influence and importance of the stream inflow can now be studied (irrespective of initial conditions) through a direct comparison with the corresponding model without a stream; the results are summarized in Table 3. The mean disk eccentricity $e_{\mathrm{d}}$ and the disk radius $r_{\mathrm{d}}$ in the final equilibrium phase are very similar in both cases, with an expected smaller disk radius and slightly smaller eccentricity due to the incoming relatively low angular momentum disk material. The surprising difference lies in the magnitude of the rate of disk precession. For the model without the stream the disk precesses in a retrograde sense quite rapidly, performing a full revolution in about 17 binary orbits. The impact of the stream is such as to slow down the precession such that it takes about 140 orbits to make a full revolution.

The surface density distribution of the model with stream inflow and an initial disk is shown during the quasi-stationary 

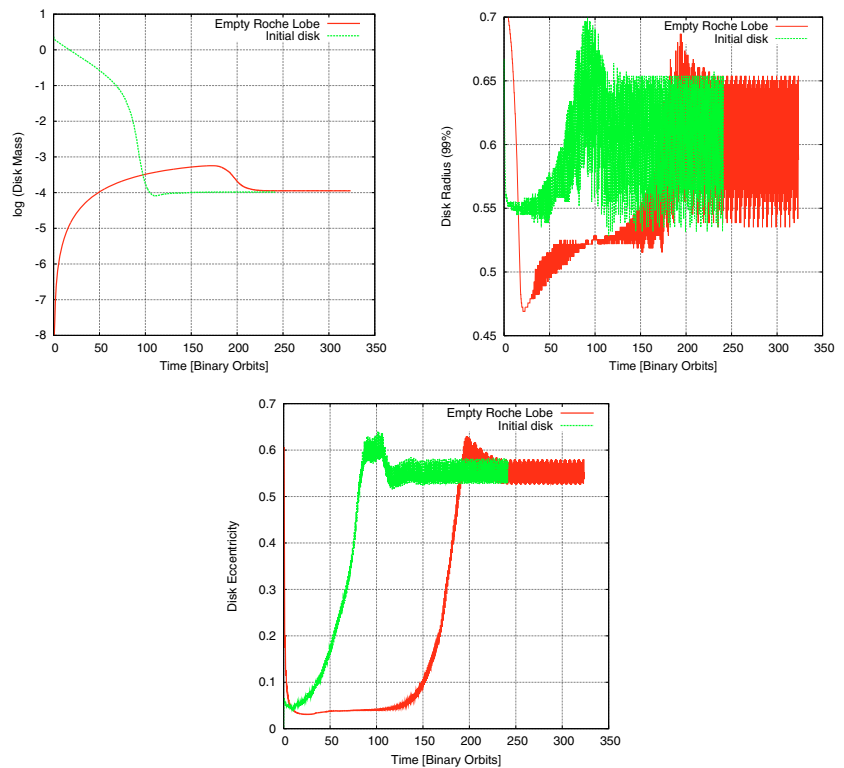

Fig. 25. Disk mass $m_{\mathrm{d}}$, radius $r_{\mathrm{d}}$ and eccentricity $e_{\mathrm{d}}$ as functions of time for models including mass inflow through the $L_{1}$ point with different initial conditions. The first (red) curve corresponds to an initially empty Roche lobe of the primary, while in the second (green) a standard initialized disk is used. The "viscous outflow" condition and a viscosity $v=10^{-4}$ (ten times standard) are used.

Table 3. Comparison of two models with and without stream inflow, all other parameters being identical. The viscosity is $v=10^{-4}$ and at the inner boundary "viscous outflow" conditions are applied. The model that has no stream corresponds to the second model in Table 2.

\begin{tabular}{llll}
\hline \hline Boundary condition & $e_{\mathrm{d}}$ & $r_{\mathrm{d}}$ & $\dot{\varpi}_{\mathrm{d}}\left[1 / P_{\text {orb }}\right]$ \\
\hline No stream inflow & 0.56 & 0.651 & -0.060 \\
With stream inflow & 0.55 & 0.610 & -0.0072 \\
\hline
\end{tabular}

phase at 4 times separated by 10 binary orbits. From Fig. 26 we can directly infer the slow retrograde precession of the disk which is also confirmed independently by a phase analysis of the $m=1$ Fourier mode at the specific radius $r=0.37$.

The change of the precession rate by about $\Delta \dot{\varpi}=0.52 P_{\text {orb }}^{-1}$ is also apparent from Fig. 27 where a quasi-stationary model with stream inflow (from Fig. 26) is continued after the stream is switched off. Clearly visible is the immediate transition to the state with no stream. The new average precession rate corresponds closely to the case with no stream inflow. Quite obviously, the disk precession rate is sensitive to modifying conditions at the outer boundary as well as at the inner boundary as we have seen above. Additional runs with cooler disks $(h=0.03$ and 0.04) demonstrate that the transition from prograde to retrograde disk precession occurs in the case with stream inflow for much larger values around between $h=0.04$ and 0.05 , see upward triangles in Fig. 14. On the other hand the disk radius and eccentricity are more robust.

\section{Conclusions}

In this paper we have performed grid-based simulations of eccentric disks in close binary systems. Adopting a gridbased rather than a particle-based method enables consideration of a wider and more appropriate range of physical parameters. We have been able to consider disk aspect ratios in the range $0.01 \leq h \leq 0.05$ and dimensionless viscosities in the

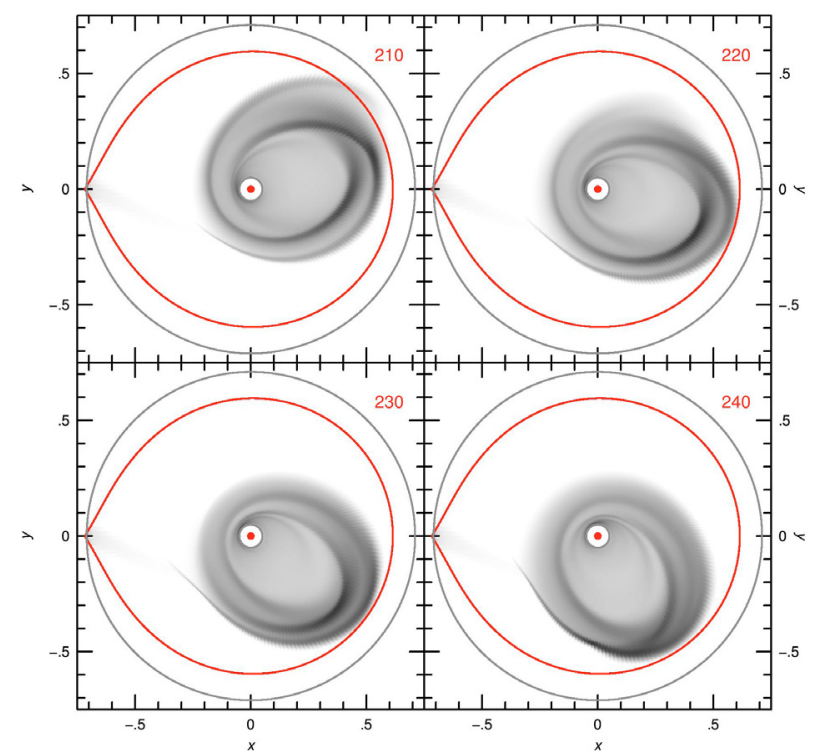

Fig. 26. Greyscale plot of the surface density of the disk for the model with stream inflow that started with an initial disk at 210,220,230 and 240 orbital periods. The solid (red) curve indicates the Roche lobe of the primary star (central red dot). The "viscous outflow" condition is used at the inner boundary.

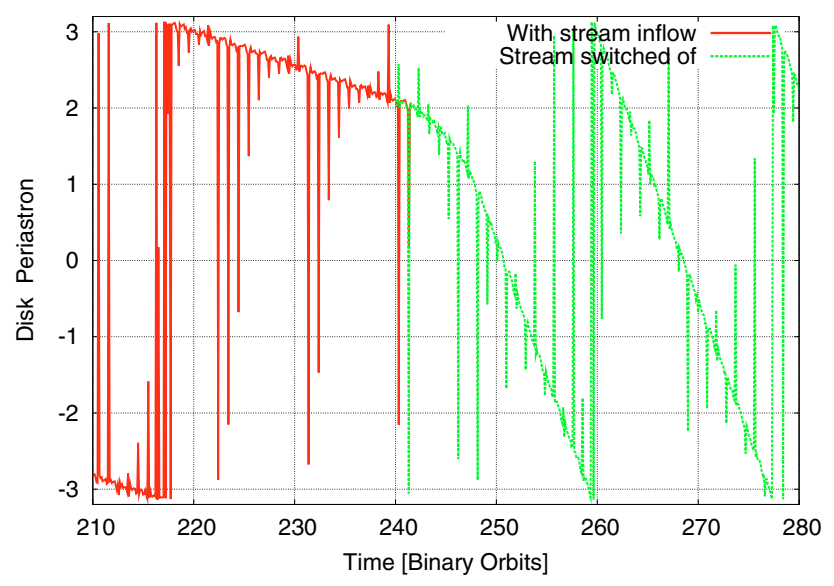

Fig. 27. Longitude of the disk pericentre (in the inertial frame) as a function of time. The solid (red) curve corresponds to a disk in a quasisteady state with stream inflow (as displayed in the previous Fig. 26). The light dashed (green) line corresponds to a model where the stream is switched off at $t=240$ and the simulation is continued.

range $3.3 \times 10^{-6} \leq v \leq 10^{-4}$, whereas particle-based methods are limited to the upper limits of these ranges. We note the relationship between $v$ and the $\alpha$ parameter of Shakura \& Sunyaev (1973) $\alpha=v / h^{2}$. Thus $v=10^{-4}$ corresponds to $\alpha \sim 0.1$ for $h=0.03$. We have also considered the effects of different inner boundary conditions, the effects of a mass-transfer stream and considered and demonstrated numerical convergence. However, due to heavy computational demands the grid-based simulations carried out in this first survey have been limited to two dimensions and simply adopted a locally isothermal equation of state. We summarize the main results of our simulations below.

\subsection{Instability to the formation of an eccentric disk}

We find instability to the formation of an eccentric disk in the mass range $0.05 \leq q \leq 0.3$. Explorative calculations have shown 
instability even for larger mass ratios up to $q=1$ in these two-dimensional simulations, see Fig. 19. The most unstable cases are those with a reflecting inner boundary, large viscosity, small aspect ratio and no mass-transfer stream. An initially non-eccentric disk quickly enters a linear phase of exponential growth of an unstable mode.

For $q=0.1$ we found growth times ranging between 162 and 15 binary orbits for $v$ in the range $3.3 \times 10^{-6}-10^{-4}$ with $h$ in the range $0.02-0.05$. A quasi-stationary precessing eccentric disk is attained in all cases in about four growth times. In the most unstable cases (large $v$ and small $h$ ) this corresponds to about 60 binary orbits or about 6 days for superoutbursting cataclysmic binaries. This is consistent with reported superhump development times of a few days.

The mean eccentricities of the quasi-stationary disks are typically in the range $0.3-0.6$ when there is no mass-transfer stream and an inner reflecting boundary. This is in the upper part of the range $0.38 \pm 0.1$ found for OY Car Hessman et al. (1992) and for IY Ma by Rolfe et al. (2001).

However, it is important to note that results are sensitive to the inclusion of a mass-transfer stream and the treatment of the inner boundary condition. For the largest viscosities, use of an inner outflow condition rather than a reflecting boundary condition reduces the final mean eccentricity from 0.5 to 0.3 . Incorporation of a mass-transfer stream does not affect the growth of eccentricity for $v=10^{-4}$ but we are able to see a transition to stability to formation of an eccentric disk at about $v=3 \times 10^{-5}$ in this case independently of the inner boundary condition. This is also consistent with the idea that the effective viscosity and consequently the mass-transfer rate through the disk is larger during the outburst phases of cataclysmic binaries during which superhumps are reported (Patterson et al. 2005).

\subsection{Quasi-steady state of the eccentric disk}

Once the simulated disks go through an instability that results in them becoming eccentric, they typically attain a characteristic quasi-steady state. This was illustrated in Fig. 10. Viewed from an inertial frame the secondary has a close approach to the slowly precessing disk apocentre approximately once every orbit, pulling out a tidal tail. This later impacts back on the disk which is not strongly affected by the secondary until the next close approach. Thus almost the entire tidal dissipation may occur as a result of these events. In this context we note that tidal dissipation has been proposed as the source of the luminosity variations during the main superhump phase while it has been proposed that effects due to modulation by the masstransfer stream become significant during the late superhump phase (Hessman et al. 1992; Rolfe et al. 2001; Patterson et al. 2005).

\subsection{Instability mechanism}

We have investigated the mechanism responsible for the excitation of the disk eccentricity and found this to be basically consistent with the mode-coupling mechanism of Lubow (1991a). Neglecting the slow precession of the disk, viewed from the inertial frame the eccentricity of the disk may be considered to arise from a stationary surface density perturbation with azimuthal mode number $m=1$. In addition the disk responds directly to the tidal potential of the companion producing a surface density response that corotates with it and consists of a sum of Fourier components with $m=1,2,3, \ldots$ with amplitudes that scale with the mass ratio, $q$. Nonlinear coupling between the response to the tidal potential and the $m=1$ mode causes the excitation of secondary density waves with pattern speeds $m \omega /(m-1)$, $m=2,3,4, \ldots$ which exceed that of the binary and are thus associated with a tidal energy and angular momentum transfer to it. The back reaction on the $m=1$ mode causes the eccentricity to grow. To work effectively the secondary waves should be associated with an inner Lindblad resonance. The most favourable case corresponds to $m=3$ and the disk gas orbiting with $\Omega=3 \omega$, at a 3:1 commensurability. In this case the Fourier component of the tidal potential with $m=3$ plays a key role.

The results of simulations with $q=0.1$ supported this view with growth being almost absent when the $m=3$ component was removed. However, for $q=0.2$ growth still occurred in this situation albeit at a reduced rate. This is apparently because higherorder couplings generated through the other Fourier components result in an effective forcing with $m=3$. Being of higher order, this is more effective at larger $q$. But it should be noted that as $q$ increases, the 3:1 commensurability is driven towards the Roche lobe thus becoming increasingly nonlinear and affected by factors such as the outer boundary condition and any stream inflow. As we indicate below, it is possible that dissipative effects not included in our two-dimensional simulations play a more important role. For these reasons we concentrated on the case $q=0.1$ in this article.

\subsection{Disk precession rates and the dependence on disk aspect ratio}

The main determinants of the disk precession rate were found to be the disk aspect ratio and the impact of a mass-transfer stream if present. Results for $0.01 \leq h \leq 0.05$ for $q=0.1$ are summarized in Fig. 14.

The quasi-steady disk precession rate $\dot{\varpi}_{\mathrm{d}}$ was found to decrease from positive values at small $h$ (prograde precession) to attain negative values at larger $h$. This is because, as expected, the pressure contribution tends to produce retrograde precession. When no mass-transfer stream was present the transition from prograde to retrograde occurred for $h \sim 0.025$. We also determined the precession rates by independently solving the linear mode problem using the average surface density profile and in view of the strong nonlinearity present obtained surprising agreement with $h$ at the transition being $\sim 0.03$.

An approximate parabolic fit to the data points for the case with no mass-transfer stream was found to be $\dot{\varpi}_{\mathrm{d}}=0.022-39 h^{2}$. This indicates that $\dot{\varpi}_{\mathrm{d}} \sim 0.02$ as indicated by observations (Patterson et al. 2005) only for $h \lesssim 0.01$. In view of the fact that values for $h$ estimated from accretion disk modelling fall in the range $0.01 \leq h \leq 0.03$ (Smith et al. 2007; Goodchild \& Ogilvie 2006), this is rather small. Note that Goodchild \& Ogilvie (2006) also obtained similarly small estimates from their one-dimensional linear formalism.

However, the simulations for which the disk was impacted by a mass-transfer stream show the transition for $h \approx 0.04$ and indicate the possibility of obtaining the observed precession rates for values of $h$ estimated from accretion disk modelling. Thus our results demonstrate the importance of the mass-transfer stream in this context as well as in the context of obtaining a transition from instability to stability at a reasonable value for the disk viscosity. On the other hand, it might be argued that the significance of the mass-transfer stream would be reduced during a superoutburst, when the accretion rate through the disk exceeds the mass-transfer rate from the companion. 
In addition we comment that there is sensitivity to the inner boundary condition such that changing from a reflecting condition to a dissipative or free outflow condition also acts to make the precession frequency move in the prograde direction.

\subsection{Comparison with particle-based simulations}

The most recent relevant SPH simulations have been performed by Smith et al. (2007). Both two- and three-dimensional simulations have been considered. Several important issues should be noted. Such simulations are inevitably highly diffusive $(\alpha \geq 0.1)$ and thick ( $h \geq 0.05$ ) so that parameter studies at smaller $h$ and smaller $\alpha$ of the type considered here cannot be carried out. Comparison has to be limited to cases with large $v$ and $h$. The SPH simulations are performed with $\sim 10^{5}$ particles and also with a mass-transfer stream. For a disk with $h=0.05$ one can estimate that there is only about one smoothing length per scaleheight in a three-dimensional simulation so that the vertical thickness is poorly resolved. The three-dimensional SPH simulations tend to produce a weaker phenomenon than that reported here with almost no activity for $q \gtrsim 0.2$ and mean disk eccentricities at the small end of the range implied by observation. In addition very long growth times approaching thousands of orbits are generally reported.

On the other hand Patterson et al. (2005) indicate that the superhump phenomenon occurs for $q \leq 0.35$ and that it is associated with high mass-transfer events during superoutbursts and a short development time requiring growth times $\sim 20$ binary orbits (see above). It may also occur in a quasi-steady manner in systems such as nova-like variables which are also believed to have a high mass-transfer rate which in this case is steady. This is also consistent with our finding that stability to eccentricity generation occurs at low viscosities and accordingly low masstransfer rates through the disk when a mass-transfer stream is present.

The reported two-dimensional SPH simulations are more in line with those reported here. This suggests that the threedimensional case is more dissipative though the adequacy of the resolution in the vertical direction should also be an issue. It is likely that inclusion of effects arising from the vertical structure of the disk could result in processes leading to additional dissipation (Papaloizou 2005) but these would need to be simulated with adequate resolution. The two-dimensional grid-based simulations reported here are possibly too unstable to forming eccentric disks, particularly at larger $q$. However, in those cases, the $3: 1$ resonance driving the instability is pushed towards the
Roche lobe where higher-order mode couplings and increasing nonlinearity occur and numerical studies should include a treatment of the vertical direction if this region is to be correctly dealt with.

Therefore an important direction for future work will be to develop three-dimensional grid-based simulations which resolve the disk vertical structure and address the issue of the importance of possible additional dissipation modes.

Acknowledgements. Very fruitful discussions with Aurelien Crida and Roland Speith are gratefully acknowledged. W.K. acknowledges the very kind hospitality and support of DAMTP during his visit to Cambridge in the spring of 2007.

\section{References}

Ak, T., Ozkan, M. T., \& Mattei, J. A. 2002, A\&A, 389, 478 Balbus, S. A., \& Hawley, J. F. 1998, Rev. Mod. Phys., 70, 1 de Val-Borro, M., Edgar, R. G., Artymowicz, P., et al. 2006, MNRAS, 370,529 Goldreich, P., \& Tremaine, S. 1979, ApJ, 233, 857

Goldreich, P., \& Tremaine, S. 1980, ApJ, 241, 425

Goodchild, S., \& Ogilvie, G. 2006, MNRAS, 368, 1123

Heemskerk, M. H. M. 1994, A\&A, 288, 807

Hessman, F. V., Mantel, K.-H., Barwig, H., \& Schoembs, R. 1992, A\&A, 263, 147

Kato, S. 1978, MNRAS, 185, 629

Kley, W. 1989, A\&A, 208, 98

Kley, W. 1998, A\&A, 338, L37

Kley, W. 1999, MNRAS, 303, 696

Kley, W., \& Dirksen, G. 2006, A\&A, 447, 369

Kornet, K., \& Rozyczka, M. 2000, Acta Astron., 50, 163

Kunze, S., Speith, R., \& Riffert, H. 1997, MNRAS, 289, 889

Latter, H. N., \& Ogilvie, G. I. 2006, MNRAS, 372, 1829

Lubow, S. H. 1991a, ApJ, 381, 259

Lubow, S. H. 1991b, ApJ, 381, 268

Masset, F. 2000, A\&AS, 141, 165

Murray, C. D., \& Dermott, S. F. 1999, Solar system dynamics (Cambridge University Press)

Murray, J. R. 1998, MNRAS, 297, 323

Ogilvie, G. I. 2001, MNRAS, 325, 231

Papaloizou, J., \& Pringle, J. E. 1977, MNRAS, 181, 441

Papaloizou, J. C. B. 2005, A\&A, 432, 757

Patterson, J., Kemp, J., Harvey, D. A., et al. 2005, PASP, 117, 1204

Rolfe, D. J., Haswell, C. A., \& Patterson, J. 2001, MNRAS, 324, 529

Schäfer, C., Speith, R., Hipp, M., \& Kley, W. 2004, A\&A, 418, 325

Shakura, N. I., \& Sunyaev, R. A. 1973, A\&A, 24, 337

Smith, A. J., Haswell, C. A., Murray, J. R., Truss, M. R., \& Foulkes, S. B. 2007, MNRAS, 378, 785

Speith, R., \& Kley, W. 2003, A\&A, 399, 395

Stehle, R. 1999, MNRAS, 304, 687

van Leer, B. 1977, J. Comp. Phys., 23, 276

Whitehurst, R. 1988, MNRAS, 232, 35

Ziegler, U. 1998, Comput. Phys. Commun., 109, 111 